\title{
The 2008 Public Release of the International Multi-tokamak Confinement Profile Database
}

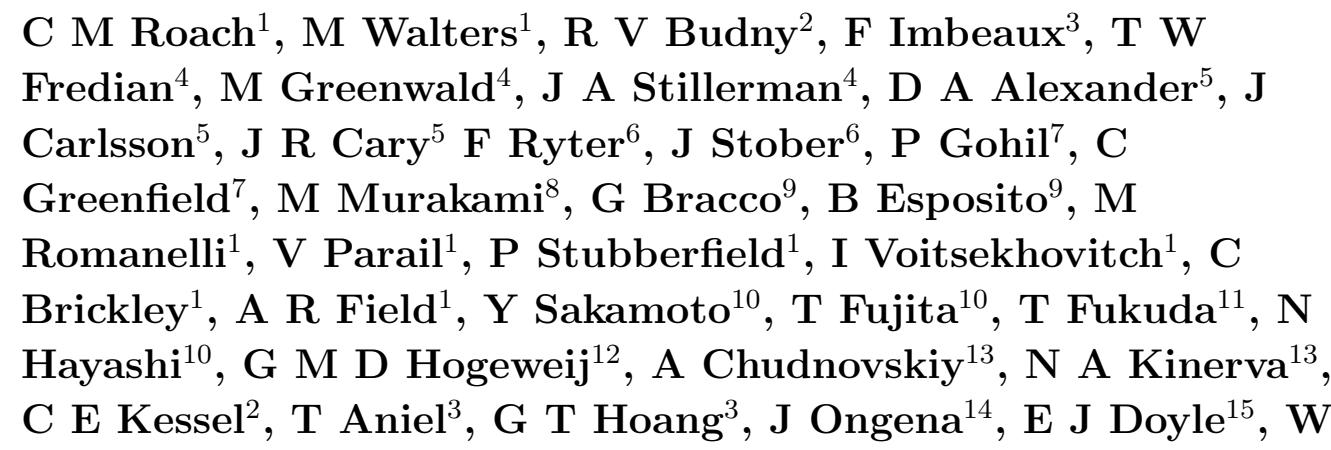
A Houlberg ${ }^{16}$, A R Polevoi ${ }^{16}$, ITPA Confinement Database and Modelling Topical Group and ITPA Transport Physics Topical Group

${ }^{1}$ EURATOM/UKAEA Fusion Association, Culham Science Centre, Abingdon, Oxfordshire, OX14 3DB, UK

${ }^{2}$ Princeton Plasma Physics Laboratory, Princeton University, Princeton, New Jersey, USA

${ }^{3}$ Association EURATOM-CEA, CEA/DSM/IRFM, CEA Cadarache, France

${ }^{4}$ Massachusetts Institute of Technology, Cambridge, Massachusetts, USA

5 Tech-X Corporation, Boulder, Colorado, USA

${ }^{6}$ Max-Planck-Institut für Plasmaphysik, Euratom Association Garching, Germany

${ }^{7}$ General Atomics, San Diego, California, USA

8 Oak Ridge National Laboratory, Oak Ridge, Tennessee, USA

${ }^{9}$ Associazione Euratom ENEA sulla Fusion, CRE Frascati, Rome, Italy

10 Japan Atomic Energy Agency, Naka, Ibaraki, Japan

${ }^{11}$ Graduate School of Engineering, Osaka University, Osaka 567-0047, Japan

12 FOM-Institute for Plasma Physics Rijnhuizen, Association EURATOM-FOM, Trilateral Euregio Cluster, the Netherlands

${ }^{13}$ Russian Research Center, Kurchatov Institute, Moscow, Russian Federation

${ }^{14}$ Ecole Royale Militaire/Köninglijke Militaire School, Association Euratom-Belgian State, Brussels, Belgium

${ }^{15}$ Department of Electrical Engineering and PSTI, University of California, Los Angeles, CA, USA

${ }^{16}$ ITER Organisation, 13108 Saint-Paul-Lez-Durance, France

\begin{abstract}
This paper documents the public release PR08 of the International Tokamak Physics Activity (ITPA) profile database, which should be of particular interest to the magnetic confinement fusion community. Data from a wide variety of interesting discharges from many of the world's leading tokamak experiments are now made available in PR08, which also includes predictive simulations of an initial set of operating scenarios for ITER. In this paper we describe the discharges that have been included and the tools that are available to the reader who is interested in accessing and working with the data. Most discharge descriptions refer to more detailed previous publications. In addition, we review physics analyses that have already made use of the profile database discharges. Public access to PR08 data is unconditional, but this paper should be cited by any
\end{abstract}


publication that makes use of PR08 data.

\section{Introduction}

The transport of heat and particles in magnetically confined tokamak plasmas has a strong influence on the viability of the tokamak approach to harnessing fusion power and, in the context of the next step ITER device, this subject has recently been reviewed extensively $[1,2]$. The International Tokamak Physics Activity (ITPA) profile database $\dagger$ is the continuation of the ITER confinement profile database [3] that was accumulated in the mid 1990s to test models of anomalous heat and particle transport in tokamaks more extensively and openly than had ever previously been attempted. The main aims were to discriminate between the ability of the models to describe present day devices, and to exploit this knowledge to improve confidence in predictions of the performance of next step fusion devices such as ITER. Initially, eleven of the world's leading tokamak experiments contributed analysed data from approximately two hundred discharges. That data included the essential physical quantities for testing 1D (radial) transport models from a broad and representative set of discharges with a range of different heating methods and in various confinement regimes (H-mode, L-mode etc). The profile database is a unique and valuable resource, providing access to multi-machine tokamak confinement data in a common format. The first release of the profile database (PR98) was made available to the public in late 1998 [4]. While no single transport model emerged as clearly superior over the full range of data $[1,4,5]$, the transport model testing exercise contributed significantly to identifying key issues and parameters, and to spurring improvements in models, codes, and our general understanding of plasma transport in tokamaks. Most of the physics-based transport models that were developed during this time predict a sharp rise in anomalous transport above a critical value of $R / L_{T}$ (where $R$ is the major radius and $L_{T}=T /(d T / d r)$ is the temperature gradient scale length), with no anomalous transport below this critical value. These so-called stiff models predict that the stored energy is sensitive to the edge temperature, and that core confinement improves substantially if the pedestal temperature can be increased. Improving our understanding of the edge physics, which determines the pedestal temperature, is presently a high priority area of tokamak research being pursued by the ITPA Pedestal and Edge Physics Topical Group. Another important area is the impact of equilibrium and turbulent plasma flows, which are capable of suppressing turbulence and improving confinement.

In 1999 the profile database moved physically from San Diego (US) to Naka (Japan), under the management responsibility of the ITPA Confinement Database and Modelling (CDBM) Topical Group. Concurrently the ITPA Transport Physics Topical Group became active in accumulating profile data from experimental discharges with internal transport barriers (ITBs). The ITPA profile database moved to its current home at UKAEA Culham (UK) in April 2001, and since then the database has both broadened in scope and undergone considerable improvements in the technology underlying its †http://tokamak-profiledb.ukaea.org.uk. 
infrastructure. The ITPA profile database is available online at http://tokamakprofiledb.ukaea.org.uk, where a website describes the data and how it is stored, and hosts various tools to facilitate accessing the data from users' physics codes. The ITB profile database, which was gathered by the ITPA Transport Physics Topical Group, was transferred to the same server at Culham in 2004. The ITB data format was harmonised with that of the ITPA profile database, allowing both datasets to be combined in the unified public release PR08.

Approximately one hundred new discharges (including the ITB profile data) have been added to the ITPA profile database since PR98 [4], and these discharges include: world record fusion power discharges from DT plasma operation in JET and TFTR; discharges with ITBs from DIII-D, JET and JT-60 Upgrade (JT-60U); high performance hybrid scenario discharges from DIII-D and JT-60U; H-mode parameter scans from ASDEX Upgrade (AUG); low aspect ratio plasmas from the Mega-Ampère Spherical Tokamak (MAST); and electron heated discharges from FTU, T-10 and Tore Supra (TS). Fuller descriptions of the experimental discharges included in this public release PR08 of the ITPA profile database are provided in Section 2. Database structure and the tools available to access the data are outlined in Section 3. The profile database presently provides a convenient way for physicists to access data from a wide range of different tokamaks, which facilitates confinement studies across machines, and Section 4 provides a brief overview of such physics analyses which have used the database. PR08 has also been used by modellers to store their predictive calculations of ITER plasma scenarios in a standard fashion, which allows key modelling assumptions to be scrutinised in detail by the broader modelling community, and these simulations are described in Appendix A.

\section{PR08 Discharges}

PR08 includes: all PR98 discharges [4]; approximately one hundred experimental discharges that have been submitted to the ITPA confinement profile database since PR98; and simulations of a number of possible ITER scenarios. The PR98 discharges were taken in the form of ASCII files from the original ITER profile database, and were converted to conform to the PR08 variables and data structures that will be described in Section 3. The ITPA profile database contains mainly processed data that have been obtained from raw measurements by the experiment analysis codes, which aim to provide the most self-consistent model of the plasma. While the database provides considerable information, it does not describe the full analysis chain. Any user of PR08 with a detailed question that cannot be answered using the contents of the database alone is encouraged to contact the person listed in the comments file who submitted the discharge. In this section we provide descriptions of the new experimental discharges in PR08, which are categorised in Table 1.

\subsection{ASDEX Upgrade}

AUG is a medium size divertor tokamak with a major radius $R$ of $1.65 \mathrm{~m}$ and minor radius $a$ of $0.50 \mathrm{~m}[6]$. The plasmas are D-shaped with typical plasma elongation $\kappa \sim 1.7$, and the shaping capabilities allow triangularity values in the range $0.15<\delta<0.4$. The six 


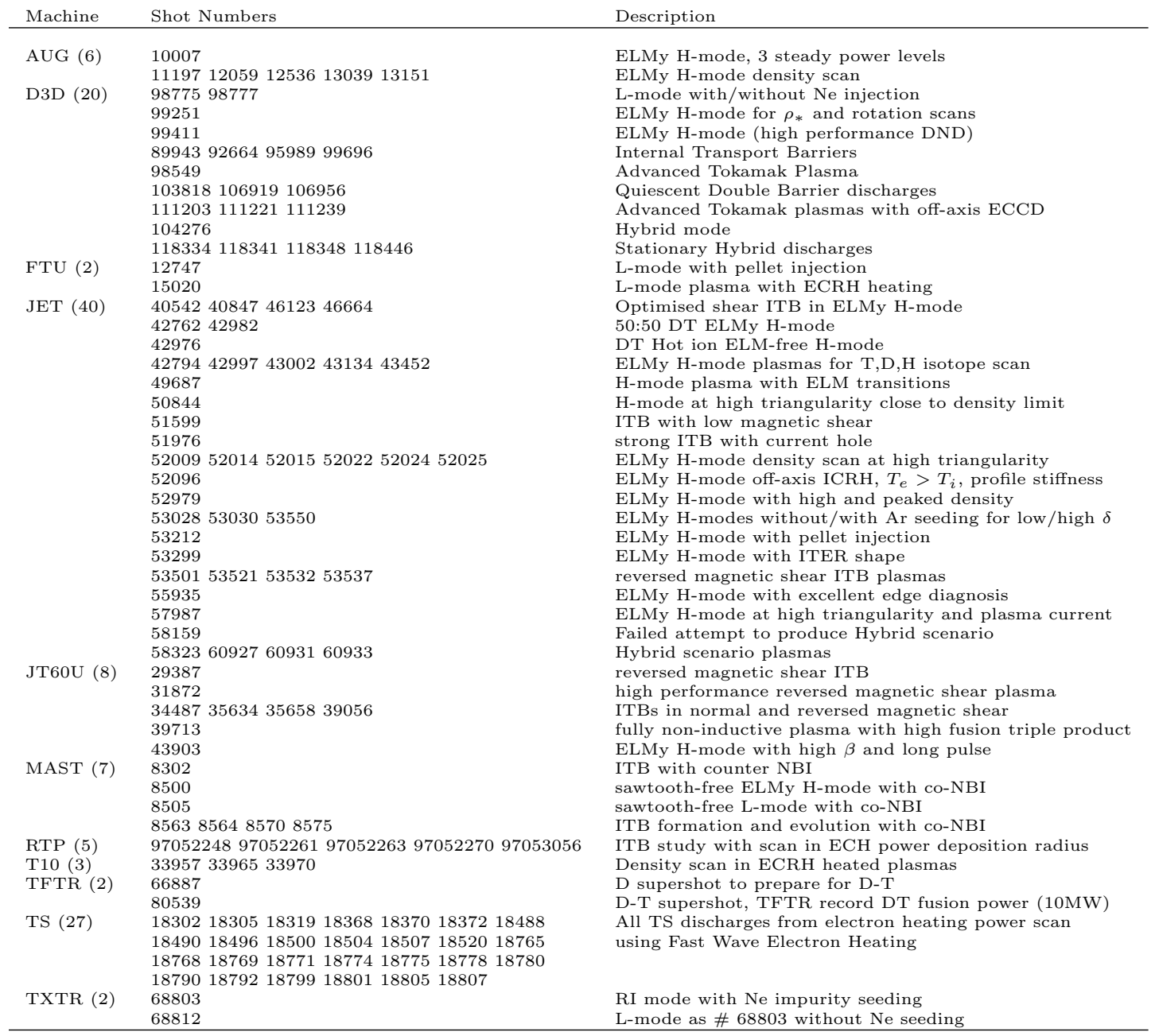

Table 1. This table lists by machine the experimental discharges in PR08 that were not included in PR98, and provides brief descriptions for each discharge. PR08 also includes simulations of ITER scenarios (described in Appendix A) and discharges from PR98, which have previously been detailed in [4].

discharges included in PR08 are deuterium plasmas heated by deuterium neutral beam injection (NBI) and are all H-modes with Type I ELMs. The average triangularity is low, with $\delta \sim 0.15$. Transport analysis of these discharges was used to compare the data with four physics-based transport models, and temperature profile stiffness was demonstrated [7]. The six discharges contributed to PR08 are representative of AUG Hmode plasmas from the time period 1998-2000: in discharge \#10007 (with plasma current $I_{p}=1 \mathrm{MA}$, safety factor at the $95 \%$ poloidal flux surface $q_{95} \approx 4$, and line averaged electron density $\bar{n}_{e} \approx 5 \times 10^{19} \mathrm{~m}^{-3}$ ) the NBI power $P_{\mathrm{NBI}}$ was decreased in steps to provide a three point scan between 7.5MW and 12.5MW; discharges \#11197, \#12059, \#12536, \#13039 and $\# 13151\left(I_{p}=1 \mathrm{MA}, q_{95} \approx 4\right.$ with $\left.P_{\mathrm{NBI}}=5 \mathrm{MW}\right)$ form a five point density scan, spanning the range $3.9 \times 10^{19} \mathrm{~m}^{-3} \leq \bar{n}_{e} \leq 7.2 \times 10^{19} \mathrm{~m}^{-3}$. Analyses of the PR08 discharges were carried out using the FPP [8] and ASTRA [9] codes for equilibrium, the ASTRA pencil code for NBI deposition and ASTRA for transport. The safety factor, $q$, profile was derived in ASTRA from current diffusion, assuming neoclassical conductivity [10] with a flat profile of effective charge, $Z_{\text {eff }}$, and without taking into account possible MHD effects. Each set of data corresponds to one steady-state time interval over which the experimental data has been averaged. 


\section{2. $D I I I-D$}

DIII-D $[11,12]$ is a highly flexible tokamak with $R=1.66 \mathrm{~m}, a=0.66 \mathrm{~m}$, and $B_{t} \leq 2.2 \mathrm{~T}$, $I_{p} \leq 3.0 \mathrm{MA}$ and $\kappa \leq 2.5$. DIII-D has a comprehensive set of diagnostic instruments, $\leq 20 \mathrm{MW}$ of NBI heating, RF heating and current drive, a pellet injector for plasma fuelling and upper and lower divertors with cryo-pumps. Twenty DIII-D discharges are included in PR08 covering most of the wide range of plasma scenarios that have been studied in DIII-D since PR98: ELMy H-modes, L-mode plasmas with and without impurity injection, internal transport barriers, quiescent double barriers, advanced tokamak plasmas including one discharge with off-axis electron cyclotron current drive (ECCD), and hybrid plasmas. All DIII-D discharges included in PR08 and described here were analysed using the TRANSP code [13], with a moments based equilibrium solver and taking the plasma boundary from EFIT [14] calculations constrained using motional Stark effect diagnostic data. NBI heating and current drive have been calculated in TRANSP using the Monte Carlo module NUBEAM [15].

Two ELMy H-mode plasmas (\#99251 and \#99411) are included in PR08. Discharge \#99251 is a counter rotating plasma (using counter-NBI) that has been used to study the dependence of confinement on plasma rotation and the $\rho_{*}$ parameter [16] (where $\rho_{*}$ is the ratio of ion Larmor radius $\rho_{i}$ to minor radius $a$ ). The temperature and density profiles and total plasma current in \#99251 closely matched those in the co-rotating (with co-NBI) ELMy H-mode discharge \#82205, which is included in the PR98 subset of PR08. Ion heat and particle transport were found to be sensitive to the direction of plasma rotation and exhibited Bohm and gyroBohm scaling in counter- and co-rotating plasmas respectively, while electron heat transport was insensitive to the sense of rotation and always exhibited gyro-Bohm scaling [16]. In a cryo-pumped double null divertor configuration (DND) using co-NBI, the other high performance ELMy H-mode discharge, \#99411, achieved a confinement enhancement factor over the ITER-89P L-mode scaling law [17] $H_{89}=2.8$ and normalised pressure parameter $\beta_{N}=3.5$ (where $\beta_{N}=\beta(\%) a(\mathrm{~m}) B_{t}(\mathrm{~T}) / I_{p}(\mathrm{MA})$ and $\beta=2 \mu_{0} p / B_{t}^{2}$ with $p$ the plasma pressure). The high performance phase in this discharge was terminated by the onset of a $2 / 1$ neoclassical tearing mode (NTM). The plasma parameters achieved in \#99411 provided suitable target parameters for the development of Advanced Tokamak (AT) discharges with negative central shear maintained through offaxis ECCD, and such scenarios, with 3MW of absorbed ECCD power, were modelled [18].

Injection of controlled quantities of light impurity gas was used to study the mechanisms for producing improved global confinement (ie the RI-mode) that has been observed in a number of tokamaks. Neon impurity injection has been demonstrated to improve confinement significantly in DIII-D L-mode discharges [19,20]. PR08 DIII-D discharges \#98775 and \#98777 were similar L-mode discharges, with and without Ne impurity injection respectively. Impurity seeding improved confinement in all channels, and gave a factor of two enhancement in energy confinement. The ion thermal diffusivity reduced to the neoclassical level, and measurements using beam emission spectrosocopy and far infrared scattering demonstrated a significant reduction in the amplitude of long wavelength turbulence [19]. Gyrokinetic microstability analysis for plasmas with impurity injection demonstrated that turbulence suppression was aided both by the enhancement of equilibrium flow shear $\omega_{\mathrm{ExB}}$ and by the reduction of mode growth rates [20]. 
Four DIII-D discharges with internal transport barriers are included in PR08. Discharges \#89943 and \#92664 demonstrated ITB formation in plasmas with reversed and strongly reversed magnetic shear in the core and with L-mode edge conditions $[21,22]$. Microstability analysis has demonstrated that $\omega_{\mathrm{ExB}}$ exceeded the maximum microinstability growth rate $\gamma_{\max }$ in the broad region of the expanding core ITB [21]. At low power, and with weak or negative magnetic shear, ITBs were found in ion temperature and toroidal angular momentum, but at higher power, as in \#92664 [22], or with stronger negative magnetic shear, as in \#95989 [23] the ITB also affected electron temperature and particle density. Microstability studies [23,24] suggested that electron temperature gradient driven turbulence (ETG) may control the electron temperature gradient in the region of the ITB, and could explain why higher electron temperature gradients were observed with stronger negative magnetic shear. In \#99696 electron cyclotron heating $(\mathrm{ECH})$ was used to heat electrons and generate a strong ITB in the electron channel, while the ions remained relatively cold $[25,26]$. Microstability analysis suggested that turbulence in this discharge was suppressed predominantly through $\alpha$ stabilisation (where $\alpha$ is the normalised pressure gradient parameter $\left.\alpha=-\left(q^{2} \beta R / p\right) d p / d r\right)$ rather than through flow shear. While ITB plasmas with L-mode edge conditions have achieved high performance in DIII-D, at high auxiliary power such discharges terminate in disruptions. Higher plasma performance was obtained by prompting an L-H transition just prior to the anticipated disruption, to combine the ITB with H-mode edge conditions. These co-NBI heated discharges proved unsustainable and also terminated in disruptions [21].

Sustained high performance plasmas combining ITB with $\mathrm{H}$-mode edge conditions were achieved using counter-NBI injection and divertor cryopumping to generate a longlived quiescent double transport barrier (QDB) [27]. The QDB mode was accessed from a quiescent H-mode (QH-mode) plasma, and PR08 discharge \#103818 [28] presents an example of the latter. In QH-mode plasmas the edge localised modes (ELMs) that arise in conventional H-mode were replaced by a continuous edge harmonic oscillation (EHO), which provided sufficient particle transport for plasma density control. In PR08 discharges \#106919 and \#106956, counter-NBI power was sufficient to supplement the QH-mode edge barrier with a core ITB and to access the QDB mode. In \#106956, a typical QDB plasma, the plasma performance parameter $\beta_{N} H_{89}$ reached a value of 7 (compared with the typical H-mode value of 4 ), and this was sustained for greater than $10 \tau_{E}$. A crucial feature of counter-NBI QDB discharges was that $\omega_{\operatorname{ExB}}$ passed through zero in the region between the barriers (where $\omega_{\mathrm{ExB}}$ is large), which prevented the core and edge barriers from coalescing $[27,29]$.

The Advanced Tokamak programme in DIII-D aims to develop the scientific basis for a steady state high performance tokamak (with simultaneous high beta, high confinement, and non-inductive current sustainment with high bootstrap fraction). Considerable progress towards long-pulse AT scenarios was made in DIII-D in co-NBI heated discharges: e.g. PR08 discharge \#98549 [30,31]. Such plasmas sustained $\beta_{N} H_{89}=9$ for $16 \tau_{E}$ [30], or $\beta_{N} H_{89}=10$ for $5 \tau_{E}$ [31]. These ELMy H-mode discharges were limited by the onset of resistive wall modes, with $\beta$ slightly above the ideal no-wall $n=1$ limit. Local heat diffusivity in these plasmas, with minimum safety factor $q_{\min }>1.5$, was similar to that in conventional sawtoothing H-mode plasmas: ion thermal transport was 2 - 3 times the 
neoclassical level. The bootstrap current fraction $f_{B S}$ reached $50 \%$, while the noninductive current fraction $f_{N I}$ reached $75 \%$ and the remaining inductive current was peaked offaxis. In later experiments the non-inductively driven current fraction $f_{N I}$ was increased further using off-axis ECCD [32,33]. In these discharges H-mode was induced early on, to achieve $q_{\min }>2.5$ at the end of the current ramp, and NBI feedback was used to sustain $\beta_{N}$. In PR08 discharges \#111203 and \#111239, 2.5MW of EC power was applied after the beginning of the high $\beta_{N}$ phase: in the former the EC injection angle drove current (ECCD), whilst radial injection in the latter discharge gave heating only $(\mathrm{ECH})$. Comparing these discharges revealed that ECCD modified the current profile at $\beta \sim 3 \%$ [32], to produce a $q$-profile with stronger negative central magnetic shear and higher $q_{0}$, which improved core confinement in all channels and raised the bootstrap current. The ECCD discharge was, however, limited by pressure driven modes associated with resistive interchange modes. With $q_{\min }>2$, \#111203 achieved and sustained over 2s: $f_{B S} \sim 0.55 ; f_{N I} \sim 0.9 ; H_{89} \sim 2.5 ; \beta_{N}=2.8 ;$ and $\beta=3 \%$ [32,33]. Delaying the application of high NBI and EC power resulted in discharges with lower $q_{0}$ and $q_{\text {min }}$. In \#111221 higher $\beta_{N}$ was accessed with $q_{0} \sim 2.1$ and $q_{\min } \sim 1.7$ [34,35], and stationary plasma profiles were maintained for 1s with: $f_{B S} \sim 0.6 ; f_{N I} \sim 0.9 ; H_{89} \sim 2.4 ; \beta_{N}=3.1$; and $\beta=3.3 \%$. While no ITB was visible in the kinetic profiles, transport analysis found higher confinement than in conventional H-mode. The stationary phase of this discharge was terminated by the onset of a small $m=5, n=3$ NTM.

DIII-D have developed feedback control systems that are crucial to sustain steady state discharges: the NBI power is controlled to maintain an approximately constant $\beta$, which is essential for operation near $\beta$ limits; and divertor pumping regulates plasma density, which is needed for current drive schemes such as ECCD. Such control systems were used in PR08 discharge \#104276, where a sawtooth-free ELMy H-mode discharge with $q_{\text {min }} \sim 1$ sustained $\beta_{N} H_{89} \sim 7$ for 6.3 s (or about $34 \tau_{E}$ or $>3 \tau_{R}$ ) [31,36]. Such hybrid mode plasmas were produced routinely on DIII-D using the following key ingredients: a broad current profile (with moderate magnetic shear and $q_{0} \sim 1$ ) was generated at the end of the current ramp using carefully timed NBI core heating during the ramp; $\beta_{N}$ was then increased, prior to the onset of sawteeth, to trigger a small $m=3, n=2$ NTM that stabilised sawteeth; and thenceforth $\beta_{N}$ was controlled using NBI feedback. Hybrid mode plasmas on DIII-D have better confinement than in conventional H-mode $\left(H_{89} \sim 2.3\right.$ compared with 2 in H-mode), and operate at close to steady state for $9 \tau_{R}$ close to the $n=1$ no wall $\beta$ limit. DIII-D have demonstrated a broad operating space for the hybrid mode: $0.35<n / n_{G}<0.7$ (where $n_{G}$ is the Greenwald density limit), $2.8<q_{95}<4.5$. Hybrid discharges \#118334, \#118341, \#118348 and \#118446 are taken from a density scan at $q_{95}=4.5$ [37], and are included in PR08. Projections of the hybrid mode of operation to ITER suggested that ITER may be able to access a wider range of high performance scenarios than were previously envisaged [37].

\section{3. $F T U$}

The FTU tokamak [38] is a circular cross-section molybdenum limiter device with $R=0.93 \mathrm{~m}$ and $a=0.3 \mathrm{~m}$. Two FTU discharges (\#12747 and \#15020) are included in PR08 and these were analysed with the EVITA transport code: the details of the 
calculation can be found in references $[39,40]$. The reconstructed $q$-profiles, obtained from the solution of the current diffusion equation assuming neoclassical resistivity, were consistent with the MHD behaviour observed in the discharges. In discharge \#12747 (with $I_{p}=0.8 \mathrm{MA}$ and $B_{t}=7.2 \mathrm{~T}$ ) two pellets were fired into the plasma core. The energy confinement time, $\tau_{E}$, in the post-pellet phase doubled with respect to that measured in identical gas-fuelled discharges (where $\tau_{E} \sim 50 \mathrm{~ms}$ ). After the peaking of the density profiles, due to the pellet fuelling, the ion heat conductivity $\chi_{i}$ fell to the neoclassical level $\left(\chi_{i} \sim 0.1 \mathrm{~m}^{2} \mathrm{~s}^{-1}\right.$ at normalised minor radius $\left.r / a \sim 0.3\right)$ and a linear relationship between $\tau_{E}$ and $n_{e}$ was recovered. More recent analyses [41] indicated that the confinement saturated at $\tau_{E} \sim 110 \mathrm{~ms}$ in pellet-fuelled discharges, as the electron heat conductivity approached the ion neoclassical value. In discharge \#15020, the ECRH heating power $P_{\mathrm{ECRH}}$ was $0.8 \mathrm{MW}$ during the current ramp-up phase, and a peak electron temperature of $\sim 12 \mathrm{keV}$ was obtained [42]; Ni and Mo dominated the impurity content. This discharge had higher $P_{\mathrm{ECRH}}$ but was otherwise very similar to \#12658, which was included in PR98 [4].

\subsection{JET}

Forty JET discharges are included in PR08, covering a wide range of confinement regimes: ELMy H-mode plasmas with broad ranges of parameters [43-57]; hybrid scenarios $[56,58,59]$; and plasmas with ITBs [60-71]. The PR08 JET discharges include examples of high performance ITER-relevant operation: H-mode scenarios at high magnetic fields and high plasma currents close to the Greenwald density limit $n_{G}$; DT plasmas with high fusion performance; and ITB discharges with high fractions of non-inductively driven plasma current and quasi-stationary operation. All JET discharges included in PR08 and described here were analysed with TRANSP [13], using its moments based equilibrium solver and taking the plasma boundary from EFIT [14] calculations. NBI heating and current drive have been calculated using the Monte Carlo module NUBEAM [15], and ICRH heating power has been modelled with the SPRUCE code [72]. The main objectives and results from each of the confinement regimes are summarised below.

\subsubsection{H-mode plasmas Most JET ELMy H-mode discharges in PR08 were dedicated} to topics of relevance to ITER, including: ITER-like plasma shapes; high density; high triangularity; high plasma current; tests of pellet injection; strong electron heating; and fusion performance in deuterium-tritium (DT) plasmas. The PR08 JET ELMy H-mode plasmas had a range of thermal ion compositions: pure deuterium; pure tritium; and mixes with similar concentrations of both deuterium and tritium.

The deuterium plasmas include a large group of discharges (\#52009-\#52025) where a density scan was performed close to the Greenwald density limit in a high triangularity single null configuration [43]. This was performed by varying the edge gas puff from zero to $4.4 \times 10^{22}$ particles $/ \mathrm{s}$. The pedestal density, $n_{e}^{\text {ped }}$, varied from approximately $6 \times 10^{19} \mathrm{~m}^{-3}$ to $1.0 \times 10^{20} \mathrm{~m}^{-3}$, reaching the Greenwald density limit. Analysis of these discharges in [43] showed that the confinement enhancement factor over the IPB98(y,2) H-mode confinement scaling law [1], $H_{98 \mathrm{y} 2}$ reduced with $n / n_{G}$ from 1.16 to 0.9 . The plasma stored energy was approximately constant (showing minor degradation) with density, while the pedestal pressure $\left(p^{\text {ped }}\right)$ clearly degraded with $p^{\text {ped }} \propto 1 / n_{e}^{\text {ped }}$. This suggested that the 
core confinement slightly increased with $n / n_{G}$ over this scan. The Type I ELM frequency increased with the gas puffing rate and $n / n_{G}$.

PR08 includes JET discharges with Ar seeding (\#53030 and \#53550) [44, 45], and a reference discharge without Ar seeding, \#53028, for comparison with \#53030. The objective of the Ar seeding was to create a radiative edge that would modify the ELM character from Type I to Type III and therefore reduce the divertor loads. Edge radiation is known to degrade the pressure pedestal, and so a further important objective was to test the impact of Ar seeding on plasma confinement. The Ar seeding experiments were performed in conditions similar to those of the density scan described above. The effect of Ar seeding on plasma performance was studied both at low triangularity $\left(\delta_{\text {lower }}=0.24\right.$, $\left.\delta_{\text {upper }}=0.18\right)$ with the X-point lying just inside the septum $\ddagger(\# 53030, \# 53028)[44,45]$, and at high triangularity $\left(\delta_{\text {lower }}=0.35, \delta_{\text {upper }}=0.48\right)$ with a plasma shape close to that proposed for ITER (\#53550) [45]. With Ar seeding in the septum configuration, high plasma density $\left(n / n_{G}=0.85\right)$ and high confinement $\left(H_{98 \mathrm{y} 2} \sim 1\right)$ were maintained after the end of the deuterium gas puff (\#53030), while without Ar seeding (\#53028) the density fell after the gas puff terminated. The discharges with Ar seeding at high triangularity (eg \#53550) obtained high $H_{98 \mathrm{y} 2}$ factors, comparable to those from similar discharges without Ar seeding [44]. High density plasmas usually have flat density profiles, but peaked density profiles were obtained both in the septum configuration discharges with Ar seeding, and in discharge \#52979 without Ar seeding (where the deuterium gas puff was set just below the level that would degrade the edge transport barrier) $[44,46]$.

Other deuterium JET ELMy H-mode discharges in PR08 include: high density plasmas with strongly peaked density profiles (\#52979) [46,47]; high density operation with ITER-like shapes (\#53299) [55]; high field side pellet injection into high density plasma close to the Greenwald limit (\#53212) [44,47]; transitions between ELM types (\#49687) [55]; medium density NBI-heated plasma with detailed edge measurements (\#55935); and off-axis ICRH experiments with $T_{e}>T_{i}$ dedicated to the study of profile stiffness (\#52096) [48].

Plasma operation with tritium is presently a unique capability of JET (though PR08 also includes tritium discharges from TFTR). The main objective in JET DT discharges was to maximise fusion performance in plasmas with a 50:50 DT mix in both standard and hot ion H-mode [49-51]. The JET contribution to PR08 includes two ELMy Hmode discharges performed at high magnetic field, plasma current, heating power and density (\#42762 and \#42982). High fusion power, $P_{\text {fus }} \sim 4 \mathrm{MW}$, and high fusion energy efficiency, $Q^{E} \sim 0.18$, were sustained over 5 s of stationary operation [49] (with $Q^{E}$ here defined as the ratio of fusion energy to plasma heating energy over the period of stationary operation, which in these discharges was limited to 5 s only by the finite duration of the heating power). DT experiments have also been performed at lower density in enhanced performance plasmas, such as hot ion ELM-free H-mode. PR08 includes a hot ion Hmode discharge in DT (\#42976) where $P_{\text {fus }}$ transiently reached a peak value of 16.1MW, corresponding to a fusion efficiency, $Q \sim 0.62[50,51]$, where $Q=P_{\text {fus }} / P_{\text {in }}$ and $P_{\text {in }}$ is the total input power.

Operating with different main ion species in JET has allowed the impact of isotope

$\ddagger$ The septum was the barrier between the inboard and outboard regions of the divertor at the time. 
mass on confinement to be studied (\#42794, \#42997, \#43002, \#43134 and \#43452) $[52,53]$. The scaling of confinement with $\rho_{*}$ was studied in dedicated experiments on JET. TRANSP [13] was used to analyse these discharges, and temperature and density profile evolution was modelled using the MMM95 transport model [52]. Experimentally the confinement was found to improve with isotope mass $M$, but the MMM95 transport model would predict confinement degradation with $M$ for fixed profile shapes, as its diffusion coefficients scale like the gyro-Bohm thermal diffusivity $\chi_{g B} \propto M^{0.5}$. The MMM95 model predictions were nevertheless consistent with the measured density and temperature profiles in hydrogen, deuterium and tritium plasmas, due to the following factors: edge pedestal temperatures (inputs to the simulations) increased with $M$; and profile shapes broadened with increasing $M$, due in part to broader neutral beam deposition profiles (arising from the slower speed of injected atoms at higher $M$ ).

2.4.2. Hybrid Scenarios Hybrid discharges are stationary high performance ELMy Hmode plasmas with reduced or fully suppressed core MHD activity $\left(q_{0}>\sim 1\right)$ and $\beta_{N}>2.5$. The hybrid regime offers the hope of improving on the plasma performance achieved in conventional H-mode. The JET hybrid discharges in PR08 [58] were produced in 2003 and include: three discharges at low toroidal magnetic field and low plasma current $\left(B_{t}=1.7 \mathrm{~T}, I_{p}=1.4 \mathrm{MA}\right)$ (\#58323,\#60927 and \#60931), and one discharge with average magnetic field and current $\left(B_{t}=2.4 \mathrm{~T}, I_{p}=2 \mathrm{MA}\right)$ and lower $\rho_{*}$ closer to the conditions of ITER (\#60933) [58]. The fusion performance parameter $H_{89} \beta_{N} / q_{95}^{2}$ reached 0.42 at $q_{95} \sim 3.9$ in JET hybrid scenarios. Stationary plasma conditions, with $35 \%$ beam driven current, $25 \%$ bootstrap current and $q_{0}>1$, were maintained for two resistive times in discharge \#58323. Transport in JET hybrid scenarios is similar to that in standard Hmode plasmas, although a systematic comparison of these confinement regimes is presently under analysis. Transport modelling of these discharges using the GLF23, Weiland and MMM95 transport models was presented in $[56,57,59]$.

2.4.3. Plasmas with Internal Transport Barriers ITBs are routinely produced in JET by shaping the current profile, leading to flat or reversed safety factor profiles in the plasma core (\#53501, \#53521, \#53532, \#53537 and \#51976) [60-71]. ITBs have also been observed in plasmas with low positive magnetic shear, where the magnetic surfaces with low-order rational q values are believed to play an important role (\#40542, \#40847, \#46123, \#46664 and \#51599) [65,66]. The usual JET technique to obtain ITBs is to apply low power NBI (or ICRH) preheat together with off-axis Lower Hybrid Current Drive (LHCD), early in the current ramp phase, so as to create a reversed shear $q$-profile by delaying the penetration of ohmic current to the magnetic axis. When this configuration has been created, the full heating power is applied. Various ITB configurations (including the optimised shear configuration with low magnetic shear, and strongly reversed magnetic shear configurations) can be produced depending on the current ramp rate, the preheat power and start time, and the power applied during the main heating phase. JET ITB discharges in several of these magnetic configurations are included in PR08.

The current density profiles in the optimised shear scenario discharges \#40542 and \#40847 were obtained by applying a low level of LHCD at the beginning of the current 
ramp, while simultaneously expanding the plasma cross-section to a full single-null Xpoint configuration [60,61], to produce high electron temperatures (up to 10keV). Delayed inward current diffusion and off-axis LHCD combined to produce strongly reversed $q$ profiles. Low ICRH preheat power of $1 \mathrm{MW}$ was applied during the current ramp in discharge \#40847. High power (18-19MW of NBI and 6MW of ICRH) was applied close to the end of the current ramp in both discharges. ITBs formed in temperature, density and momentum during the high power heating phase, first in the plasma core, and then expanding rapidly outwards to $\rho \sim 0.67$ (where $\rho$ is the normalised square root toroidal flux). ITBs formed in plasmas with an L-mode edge, and subsequent transitions to Hmode have added an edge pedestal to the persisting ITB. Discharge \#40847 was not stationary, and plasma performance improved continuously until the first Type I ELM degraded confinement. Pressure peaking in plasmas with ITBs has often led to internal kink modes and subsequent disruptions (eg \#46664 [62]). Nevertheless, high performance has been sustained in optimised shear scenarios for a few confinement times by varying the heating power and ELM behaviour (\#40542 [60]).

In two of the discharges described above, ITBs in temperature, density and momentum formed together initially in the region of weakly reversed shear close to the location of the safety factor minimum, $q_{\text {min }}$. The ITB positions then expanded gradually while the position of $q_{\text {min }}$ remained approximately constant. Transport modelling with the ASTRA code [9] has demonstrated that the outward expansion of the ITBs in these discharges correlates well with the position of the maximum equilibrium ExB flow shear $[63,64] \S$. These observations were used to develop a transport model including the stabilising effects of the ExB shear and magnetic shear, which was then applied to model temperature and density profile evolution in discharges \#40847 and \#40542, and in a number of DIII-D and TFTR reversed shear discharges that were included in PR98 [63,64]. This model provided a satisfactory description of the ITB dynamics in these discharges, and successfully described the ITB formation and expansion towards the edge.

Discharge \#51976 is an example of the current hole configuration with strongly negative magnetic shear in the plasma core [66-68]. Reconstruction of the $q$-profile in this discharge is described in [67], where transport and microstability analysis, using Weiland and Rogister [74,75] models and the TRB code [76], were also presented. The TRB code predicted that negative magnetic shear, close to that observed in \#51976, should stabilise trapped electron modes (TEM) but have little impact on ion temperature gradient driven modes (ITG). This could explain an electron ITB, but not the strong ion ITB observed in experiment. Calculations with the flux-tube gyrokinetic code GS2 predicted turbulence stabilisation under these conditions [68].

Discharge \#53521 is a good example of a nearly stationary ITB discharge with a high non-inductive current fraction. Improved confinement was maintained for close to the resistive time $\tau_{R}$, which is much longer than the energy confinement time $\left(\tau_{R} \sim 37 \tau_{E}\right)$ [69]. A high non-inductive current fraction (nearly 80\%) allowed the ITB to be sustained for up to $11 \mathrm{~s}$ in plasmas with an H-mode edge and Type III ELMs. ITBs were observed

$\S$ Poloidal rotation of the main ion species, which is needed to determine the full ExB flow shear, was modelled using ASTRA with NCLASS [73] as measurements were not available. 
in electron and ion temperature, density and toroidal rotation. Both ExB shear and reversed magnetic shear played an important role in ITB formation and sustainment in this discharge. Stability analysis has shown that the ExB shear exceeds the growth rate of ITG modes in the barrier region, while reversed magnetic shear plays an important role reducing microinstability growth rates. The importance of the non-monotonic $q$ profile has also been illustrated by the rapid recovery of the ITB after a number of spontaneous collapses of the ITB during the discharge. These collapses affected pressure, toroidal rotation and ExB shear profiles on a fast time scale, but not the $q$-profile, which maintained its non-monotonic shape.

Two discharges have addressed the impact of electron heating on ITBs. These discharges had similar LHCD preheat and current drive, but different heating methods were applied during the current flat-top. NBI heating in \#53532 was partly replaced by hydrogen minority ICRH heating in \#53537. Stronger electron heating in the latter case did not lead to degradation of ion confinement with the increase of $T_{e} / T_{i}$ [70], with the possible explanation that the electron temperature gradient was insufficient to excite TEM turbulence [70].

The formation of electron ITBs during the early current ramp phase in plasmas with $T_{e} / T_{i}>1$ and in the absence of external momentum input, was investigated in discharge \#53501 where LH waves were launched during the current ramp phase for electron heating and non-inductive current drive [71]. The barrier formed just inside the location of $q_{\min }$ and slowly moved inward. This inward displacement of the electron ITB followed the inward motion of the reversed shear region.

\subsection{JT-60U}

JT-60U [77-79] is a large tokamak with $R \leq 3.5 \mathrm{~m}, a \leq 1.1 \mathrm{~m}, B_{t} \leq 4.4 \mathrm{~T}, \kappa \leq 1.8$ and $\delta \leq 0.6$. A variety of auxiliary heating methods, such as positive ion based neutral beam injection, P-NBI, (2 units of co-NBI, 2 units of counter-NBI, 7 units of perpendicular NBI), negative ion based neutral beam injection, N-NBI, lower hybrid, LH, and electron-cyclotron, EC, allow flexible control of heating, rotation and current drive profiles. All JT-60U discharges included in PR08 were performed with a Wshaped semi-closed divertor configuration that enhanced the control of heat, fuel particles and impurities in the divertor region. The ranges of parameters covered in the PR08 discharges from JT-60U are $3.1 \mathrm{~m} \leq R \leq 3.4 \mathrm{~m}, 0.74 \mathrm{~m} \leq a \leq 0.87 \mathrm{~m}, 1.7 \mathrm{~T} \leq B_{t} \leq 4.3 \mathrm{~T}$, $0.53 \mathrm{MA} \leq I_{p} \leq 1.8 \mathrm{MA}, 1.4 \leq \kappa \leq 1.77$ and $0.05 \leq \delta \leq 0.37$, while the heating power is in the range $2.7 \mathrm{MW} \leq P_{\text {aux }} \leq 25.5 \mathrm{MW}$. All JT-60U discharges were analysed using the 1.5-dimensional transport code TOPICS (TOkamak Prediction and Interpretation Code System) $[80,81]$, which reconstructed MHD equilibria using constraints from a motional Stark effect (MSE) diagnostic [82]. Heating and current drive from neutral beam injection and from electron cyclotron waves were respectively calculated using the OFMC (Orbit Following Monte Carlo) [83] and EC-Hamamatsu [84] codes. Eight JT-60U discharges are included in PR08: three high $\beta_{p}$ ELMy H-mode discharges \#34487, \#39713 and \#43903 (where $\beta_{p}=2 \mu_{0} p / B_{p}^{2}$ and $B_{p}$ is the poloidal magnetic field strength at the plasma edge); and five reversed magnetic shear discharges \#29387, \#31872, \#35634, \#35658 and \#39056. 
A transition (or 'ITB event') from a 'weak' parabolic-type to a 'strong' box-type ITB was observed in discharge \#34487, under ELMy H-mode edge conditions. Three time slices (right before the transition, just after the transition, and after the ITB has become well developed) demonstrated the non-local nature of transport at the transition $[85,86]$. There are two high $\beta_{p}$ ELMy H-mode discharges, with P-NBI, N-NBI and EC heating. In the first discharge \#39713 [87], the record fusion triple product under fully non-inductive current drive was achieved, where the non-inductive current fraction was enhanced by the injection of N-NBI and EC. The values of collisionality and $\beta_{N}$ were close to those in ITER. The second discharge \#43903 [88] was in the hybrid regime (relevant to ITER hybrid operation), where high $\beta_{N} \sim 2.5$ was sustained for $15.5 \mathrm{~s}$ with feedback-control to maintain constant $\beta$.

There are five discharges for the study of box-type ITB formation in reversed magnetic shear plasmas with different $I_{p}$ at high $B_{t}(3.6 \mathrm{~T}-4.3 \mathrm{~T})$ (discharges \#29387, \#31872, \#35634, \#35658 and \#39056). The ITB formation condition and the transport characteristics for heat and particles were investigated using linear stability analysis with the profiles from \#39056 (4 time slices: before and just after the initial ITB formation, with a strong ITB, and later with a very well-developed strong ITB) [89]. Linear microinstability growth rates remained positive in the strong ITB region, even including the stabilising effects of sheared ExB rotation, indicating incomplete stabilisation of microinstabilities. The ratio of electron convective heat flux to electron conductive heat flux was calculated from the quasi-linear particle and heat fluxes from the dominant microinstability for well-developed strong ITB profiles. This ratio was estimated to be close to 2.5 in the ITB region, and dropped to a slightly negative value outside the ITB. The experimental profile of this ratio showed similar trends to the calculated profile.

\section{6. $M A S T$}

MAST is a spherical tokamak of aspect ratio $R / a \sim 1.3, R \sim 0.85 \mathrm{~m}, I_{p} \leq 1.3 \mathrm{MA}$, $B_{t} \leq 0.58 \mathrm{~T}$, and is typically operated in a DND configuration at an elongation $\kappa \sim 2$. The MAST vacuum vessel is external to the poloidal field coils, offering good access to the plasma for profile and imaging diagnostics. The MAST PR08 plasmas were heated by two tangentially directed NBI systems, delivering $P_{\text {inj }} \leq 3.3 \mathrm{MW}$ with D injection at $53 \mathrm{keV}$ energy.

Transport and NBI analyses were performed using TRANSP, with the EFIT equilibrium code providing the plasma boundary and initial $q$ profile and subsequent evolution of the current profile being calculated in TRANSP from poloidal field diffusion [90]. Ion temperature $\left(T_{i}\right)$ and toroidal rotation $\left(v_{\phi}\right)$ profiles were available from CXRS at the time of a fast NBI cut-off and the profile data from a time $5 \mathrm{~ms}$ earlier. Time resolved electron temperature $\left(T_{e}\right)$ and density $\left(n_{e}\right)$ profiles were measured with a $200 \mathrm{~Hz}$ Nd-YAG TS system. The profile evolution of $T_{i}$ and $v_{\phi}$, required for time-dependent transport analysis, was obtained by assuming a constant profile of $T_{i} / T_{e}$ and from the density profile asymmetry respectively. $Z_{\text {eff }}$ profiles were also obtained from a $2 \mathrm{D}$ bremsstrahlung imaging diagnostic. PR08 includes seven NBI heated discharges with $I_{p}=800 \mathrm{kA}$, encompassing sawtooth-free L-mode and H-mode regimes, and co- and counter-NBI heated ITB scenarios [91]. In all discharges one beam source used D, and 
the other H to improve CXRS measurements through the greater beam penetration of $\mathrm{H}$.

The sawtooth-free L-mode and H-mode discharges [91] were produced by applying $1.8 \mathrm{MW}$ of NBI during the initial $4.7 \mathrm{MA} / \mathrm{s}$ current ramp from $0.1 \mathrm{~s}$, so that the central safety factor $q$ remained above 1 until the onset of the first sawtooth. Discharge \#8500 was run in a connected double-null divertor configuration (C-DND) with mid-plane high field side fuelling to induce a transition to an ELMy H-mode at 0.16s. Conversely, the L-mode discharge, \#8505, was produced in an unbalanced L-DND configuration with gas puff fuelling from the low field side to suppress the onset of H-mode. At the time of the NBI cut-off at $0.29 \mathrm{~s}$ the central electron temperature $T_{e} \sim 1-1.2 \mathrm{keV}$ in both the L-mode and $\mathrm{H}$-mode discharges and the line-average density was similar $\bar{n}_{e} \sim 4 \times 10^{19} \mathrm{~m}^{-3}$. The H-mode discharge had more strongly peaked $T_{e}$ and flatter $n_{e}$ profiles than in the L-mode discharge. The ion temperature, which peaked around $1.3 \mathrm{keV}$, was higher in L-mode, and had a steep-gradient region in both confinement modes between $0.4<r / a<0.6$. In both discharges the toroidal Mach number is high $\left(M_{\phi} \leq 0.4\right)$. The H-mode plasma had a stored energy $W_{\mathrm{pl}} \leq 95 \mathrm{~kJ}$ which was $\sim 20 \%$ higher than in L-mode, and a higher thermal energy confinement time of $35 \mathrm{~ms}$ compared to $24 \mathrm{~ms}$ in L-mode. Microstability analysis for \#8500 and \#8505 reported in [92] found that $\omega_{\mathrm{ExB}}$ generally exceeds the growth rates of microinstabilities with $k_{\perp} \rho_{i}<O(1)$ (including ITG modes), where $k_{\perp}$ is the perpendicular wavenumber, and is smaller than the growth rates of modes with $k_{\perp} \rho_{i} \gg O(1)$ (including ETG modes). Including magnetic perturbations was found to be significant at mid-radius in these equilibria, where locally $\beta \sim 0.1$ : at $k_{\perp} \rho_{i}<O(1)$ strongly electromagnetic modes dominated over ITG modes, and these exhibited tearing and twisting parity in the H-mode and L-mode plasmas respectively; and including magnetic perturbations had a stabilising influence on ETG instabilities [92]. Numerical experiments to probe the drive mechanism for the microtearing mode found that the growth rate is sensitive to the electron temperature gradient, magnetic drifts, and collisionality [93]. Nonlinear electron scale microturbulence calculations for a typical MAST H-mode plasma suggested that significant electron heat transport can be carried by ETG turbulence [94].

By applying 1.8MW of NBI heating from the start of the current ramp to a large radius and low-density target plasma, it was possible to optimise the formation of ITBs in L-mode plasmas to produce reversed magnetic shear and high toroidal rotation with $M_{\phi} \leq 1$. With counter-NBI heating the driven ExB flow shear augmented that due to the pressure gradient, producing a broader ExB shear profile. In discharge \#8302 [91], at the time of NBI cut-off $(t \sim 0.195 \mathrm{~s})$ the profiles exhibited a strong ITB in the electron channel with $R / L_{T} \sim 20$, and in later discharges with high-resolution CXRS measurements it was established that the ITB was also present in the ion channel. At the ITB the electron thermal diffusivity $\chi_{e}$ approached the ion neoclassical level. With co-NBI heating, ITBs were also formed in the ion channel and to a lesser extent in the electron channel. Discharges \#8563, \#8564, \#8575 and \#8570 form a series of similar discharges with the NBI cut-off at $t=0.90 \mathrm{~s}, 0.145 \mathrm{~s}, 0.195 \mathrm{~s}$ and $0.245 \mathrm{~s}$ to provide profile evolution measurements [91]. In discharge \#8575 a strong ion ITB with $R / L_{T} \sim 15$ was observed at $t=0.195 \mathrm{~s}$ and at mid-radius $r / a \sim 0.5$, where the ion thermal diffusivity $\chi_{i}$ approached the neoclassical ion thermal diffusivity $\chi_{i}^{n c}$. A weaker electron ITB also formed at the same location with $R / L_{T} \sim 15$ with a corresponding reduction in electron 
thermal transport (with $\chi_{e} \sim 2-3$ times the ion neoclassical level). The ITBs formed in the plasma core and evolved outwards, eventually being degraded by the onset of MHD activity.

\subsection{RTP}

The RTP tokamak was a medium-size circular limiter device with $R=0.72 \mathrm{~m}, a=0.164 \mathrm{~m}$, $I_{p}=150 \mathrm{kA}, B_{t}=2.4 \mathrm{~T}$, and pulse duration up to $0.6 \mathrm{~s}$. Auxiliary heating was provided by one gyrotron, delivering $350 \mathrm{~kW}$ 2nd harmonic X-mode ECRH power, deposited on- or off-axis in a very narrow region $(<0.1 a)$. RTP was equipped with $T_{e}$ and $n_{e}$ diagnostics with excellent radial and time resolution, but no $T_{i}$ diagnostic was available. However, due to the weak electron-ion coupling, RTP discharges are well suited for studying electron thermal transport. A locally developed code was used for equilibrium and transport analysis of the RTP PR08 discharges. At the time when the profiles were measured, many current diffusion times after thermal equilibrium, the current density profile could be assumed to be fully relaxed. The $q$-profiles were calculated from the measured $T_{e}$ and $n_{e}$ profiles, assuming neoclassical resistivity, a flat $Z_{\text {eff }}$ profile, and including bootstrap current. The $T_{i}$ profile was estimated by assuming a model for the ion thermal diffusivity $\chi_{i}=3 \chi_{i}^{n c}$, and the typical core ion temperature estimate $T_{i}(0) \sim 0.4 \mathrm{keV}$ agreed well with scarce neutral particle analyser measurements. In the given experimental conditions, the TORAY code [95] found that nearly $100 \%$ absorption of ECRH was obtained in a single pass. The modelled width of the ECRH power deposition profile was narrow, due to the excellent focussing of the launcher, and in transport analysis this profile was reasonably approximated as a Gaussian with full-width-half-maximum $\sim 0.1 a$.

The dependence of confinement on the normalized ECRH power deposition radius $\left(\rho_{\text {dep }}\right)$ was studied systematically in RTP by scanning $\rho_{\text {dep }}$ in steps of $\sim 1 \%$ in a series of otherwise similar discharges. It was found that the $T_{e}$ profile did not adapt gradually to the changing heating location: instead, sharp transitions between a finite number of discrete profiles (corresponding to a discrete set of levels for the central electron temperature) were observed. Those sharp transitions corresponded to changes in the $q$ profile, where $q_{\min }$ jumped across a low-order rational value, eg $1,4 / 3,3 / 2,2,5 / 2$, and 3 . This indicated that $\chi_{e}$ was a direct function of $q$, with transport barriers occurring near low-order rational values of $q$ and with high values of $\chi_{e}$ elsewhere [96,97]. Five medium density discharges representative of the main $T_{e}$ profile levels, from $q_{\min }<1$ to $q_{\min }>3$, are included in PR08 (shots \#97052248, \#97052263, \#97052261, \#97053056 and \#97052270). With far off-axis ECRH $\left(\rho_{\text {dep }}=0.4\right)$, a regime with hollow $T_{e}$ and reversed magnetic shear inside $\rho_{\text {dep }}$ was obtained. For these cases the electron power balance inside $\rho_{\text {dep }}$ yields $\chi_{e}$ close to, or even below, zero, indicating the existence of an outward convection term [98, 99]. An example of such a discharge at high density is included in the database, together with an ohmically heated reference discharge (shots \#96040237, \#96040238).

The paleoclassical transport model [100] has been extensively tested against the data from the ECRH $\rho_{\text {dep }}$ scan in RTP, as represented by 5 discharges in the database. Excellent qualitative agreement with experimental results was reported [101]. 


\section{8. $T-10$}

T-10 is a limiter tokamak with circular cross-section, $R=1.5 \mathrm{~m}$, and $a \sim 0.3 \mathrm{~m}$. ECRH ( $P_{\mathrm{ECRH}}$ up to $2.6 \mathrm{MW}$ ) is used for plasma auxiliary heating and current drive. The PR08 discharges from T10 were analysed using the ASTRA code [9], using the measured density and electron temperature profiles and experimental measurements of $Z_{\text {eff }}$ and boundary loop voltage (VSURF). The ion temperature profile was estimated by assuming that ion heat transport was close to the neoclassical level, with the ion thermal diffusivity $\chi_{i}$ modelled using $\chi_{i}=2.3 \chi_{i}^{n c}$ with ECRH heating, and $\chi_{i}=\chi_{i}^{n c}$ in the ohmic heating phase. The resulting modelled neutron yield and stored energy agreed well with experimental measurements. In the submitted 0-D data: the absolute $\beta_{p}$ variable, BEPDIA, was taken from diamagnetic measurements, the Ohmic power POHM was given by $I_{p} \times$ VSURF, and the radiated power PRAD contains the total radiation losses measured by pyroelectric bolometer from both inside the limiter area and in the limiter shadow area. In the 2D data: the Ohmic power density, QOHM, was calculated using the experimental electron temperature, effective charge, $Z_{\text {eff }}$, which was taken as constant across the plasma crosssection, and neoclassical conductivity; and WTOT was calculated using the experimental electron temperature and density profiles and the modelled ion temperature profile.

T-10 experiments with ECRH have demonstrated regimes where the energy confinement time, $\tau_{E}$, increases linearly with density for $n_{e} / n_{G}<0.6$, and saturates for $n_{e} / n_{G}>0.6$ [102]. The T-10 contribution to PR08 consists of three discharges from a plasma density scan in such a regime, where the density spanned the range $0.25 \leq n / n_{G} \leq 1$ : in \#33957 (low density) $\tau_{E}$ was in the linear regime; in \#33970 (high density) the $\tau_{E}$ density dependence had saturated; and in \#33965 the density was close to the intermediate transition point between these states $[102,103]$. On-axis 2nd harmonic ECRH (140GHz, X-mode) provided auxiliary plasma heating, and these waves were launched along the major radius from the low field side to give an absorbed ECRH power of $0.9 \mathrm{MW}$ during this scan. Fluctuation measurements, made on T10 using correlation reflectometry, were used to investigate how the underlying turbulence evolved with increasing plasma density [104]. At low density, low frequency $(\sim 100 \mathrm{kHz})$ quasicoherent oscillations, attributed to ion temperature gradient driven turbulence, were found to dominate the turbulence spectra, while at higher density, the spectra became dominated by higher frequency $(\sim 200 \mathrm{kHz})$ quasi-coherent oscillations, attributed to trapped electron mode turbulence [104].

\subsection{TFTR}

TFTR was a large limiter tokamak with almost circular cross section, $R=2.6 \mathrm{~m}$ and $a=0.9 \mathrm{~m}$. Most PR08 discharges were heated by neutral beams (up to 40MW) usually with deuterium, but also with tritium during the DT campaign. Two of the beam injectors were oriented in the co-current direction and two in the counter direction, allowing a wide range of co and counter NBI heating fractions to be investigated. 107 TFTR Ohmic, Lmode and supershot discharges were included in the PR98 release of the profile database, and these were described in [4]. The supershots were produced by lowering the wall recycling by extensive wall conditioning or by coating the walls with lithium. They had 
high energy confinement relative to L-mode. Some PR98 submissions have been updated for PR08 using more recent TRANSP analyses with an upgraded neoclassical module [105] and improved equilibrium solvers (such as VMEC [106], ESC [107], and TEQ [108]).

Two additional TFTR discharges are included in PR08. The first (\#66787) was a reproducible DD supershot that was developed to prepare for alpha heating experiments. The normalised pressure, $\beta_{N}=1.95$ and the Greenwald fraction was 0.5. Transport modelling (using TRANSP) of this supershot and extrapolations to an analogous DT supershot were presented in [109]. The second discharge(\#80539) included PR08 is the DT pulse that achieved the TFTR and world record tokamak DT fusion power of 10.3 MW, which has since been surpassed only in JET. The auxiliary heating was 25.0MW of tritium neutral beam injection and $14.6 \mathrm{MW}$ of deuterium neutral beam injection. The normalised pressure $\beta_{N}=1.85$, and the peak value of $Q_{D T}=0.26$ (where $Q_{D T}$ is defined as $P_{\mathrm{DT}} / P_{\text {ext }}$ and $\left.P_{\text {ext }} \equiv P_{\text {NBI }}+P_{\text {Ohmic }}\right)$. After the high performance phase with a Greenwald fraction 0.5 , this discharge had a minor disruption leading to a carbon bloom and a corresponding increase in the electron density to a Greenwald fraction of one. Transport modelling of this discharge was described in [51].

\subsection{TS}

Tore Supra is a circular limiter device with $R=2.4 \mathrm{~m}$ and $a=0.8 \mathrm{~m}$ [110]. The Tore Supra discharges included in PR08 are from a series of L-mode shots heated by Fast Wave Electron Heating [111] (FWEH). This heating scheme allows pure electron heating, localised in the very core of the plasma, without toroidal momentum input. In these PR08 discharges the diagnostic data was mapped onto a simple flux surface geometry that was deduced from the magnetic measurements, which provided the Shafranov shift and an estimate of the current profile peaking. This procedure is reliable for the circular geometry of Tore Supra, and no equilibrium code is required. The Fast Wave power deposition profile has been computed with the codes ALCYON [112] and PION [113], and this is localised inside $r / a<0.2$ with more than $90 \%$ of the power absorbed by electrons. These hot electron plasmas are characterised by weak electron-ion collisional coupling. The PR08 Tore Supra shots consist of sawtooth-free helium plasmas with a scan of total FWEH power between 1.5MW and 7.5MW with the same electron density and $q$ profiles, at $I_{p}=0.65 \mathrm{MA}$ and $B_{t}=2.2 \mathrm{~T}$. These discharges were used to demonstrate a critical inverse temperature gradient length $\left(R / L_{T}\right)_{\text {crit }}$ for electron heat transport [114-116].

\subsection{1. $T X T R$}

TEXTOR is a medium size tokamak $(R=1.75 \mathrm{~m})$ with circular cross-section, which is equipped with two poloidal limiters and one toroidal limiter. The minor radius of TEXTOR discharges can vary between $a=0.46 \mathrm{~m}$ and about $0.4 \mathrm{~m}$, depending on the positioning of the poloidal and/or toroidal limiters. Auxiliary heating on TEXTOR consists of NBI, ICRH and ECRH. Two neutral beam injectors are installed, one co-beam and one counter-beam, capable of delivering up to $2 \mathrm{MW}$ at a maximum of $60 \mathrm{kV}$ each. The ICRH system consists of two antenna pairs, fed by two separate generators capable of delivering $2 \mathrm{MW}$ each. The ECRH system consists of one $140 \mathrm{GHz}$ gyrotron, delivering 
up to $0.8 \mathrm{MW}$ in 2 nd harmonic X-mode. The toroidal magnetic field is $B_{t} \leq 2.6 \mathrm{~T}$ and the plasma current $I_{p} \leq 520 \mathrm{kA}$. The maximum pulse duration was originally $\sim 2 \mathrm{~s}$, but in 1994 the tokamak was upgraded to allow for pulses up to 10s: since then the machine has officially been called TEXTOR-94. TRANSP [13] was used for equilibrium and transport analysis and to verify data consistency in the PR08 TEXTOR discharges. NBI and ICRF power deposition profiles were calculated using NUBEAM [15] and SPRUCE [72], respectively.

After the upgrade of TEXTOR the Radiative Improved (RI) mode was developed. The RI-mode was created with a strongly radiating edge, sustained by controlled Ne or Ar puffing. Its confinement recovered the favourable neo-Alcator density scaling $[117,118]$. Consequently, the RI-mode allows one to operate high-confinement discharges at a density close to the Greenwald limit. A pair of discharges, one in RI-mode (\#68803) and one in L-mode (\#68812), have been included in PR08.

Microstability of the two TEXTOR discharges \#68803 and \#68812 was analysed with the electrostatic linear gyro-kinetic code KineZero [119], which showed that impurity seeding was globally stabilising and responsible for triggering the profile peaking. The consequent increase of flow shear was found to maintain the improved confinement.

\section{Database Description, Access and Associated Tools}

PR08 has evolved naturally from the PR98 release of the international multi-tokamak confinement profile database that has been described previously [4]. In addition to the new PR08 discharges (see Section 2), considerable improvements have been made in several important aspects of the database infrastructure: (i) the structure and nature of the data itself; (ii) technology as to how the data is stored and made accessible; and (iii) tools for more conveniently working with the data. In this section we describe the major new developments.

\subsection{Nature and Structure of the Data}

Data structure and content in PR08 are determined by the principal goal of the database being to study the radial transport of heat and particles in tokamak plasmas. The data structure remains fully consistent with that described in detail in [4], to which we refer the reader for detailed descriptions of the original variables and data structure. Here we focus on recent modifications, which have generally been minor but nevertheless significant. As a guiding principle, we have aimed to maximise backward compatibility to reduce work for existing profile database users. As described in [4], the profile database stores four types of data for each discharge: comments data describing the discharge, generally in the form of text; 0D data consisting of the values of a number of global quantities at a small number of key time points in the discharge; 1D time trace data giving a more complete time evolution for such quantities; and 2D data allowing for the storage of radial profiles as functions of time. All 2D profiles are stored as functions of the radial flux surface label $\rho$ (where $\rho$ is the normalised square root toroidal flux).

In the transition from PR98 to PR08 changes were made to the 0D, $1 \mathrm{D}$ and $2 \mathrm{D}$ variable definitions, leaving the comments variable definitions unaltered. The most 
substantial changes have been to the $0 \mathrm{D}$ variable definitions, and the original list of 78 0D variables has been extended to 144 . Most of the additional 0D variables were defined by the ITPA Transport Physics Topical Group to characterise discharges with ITBs. The new data format sets the standard for all ITPA profile databases. Other changes include:

- A larger number of fast and thermal ion species can be accommodated in PR08, and these are now unambiguously identified. Thermal ion densities are stored in the 2D variables $\operatorname{NM} x(1<x<9)$ and their corresponding atomic and mass numbers are stored in $0 \mathrm{D}$ variables $\mathrm{NM} x \mathrm{Z}$ and $\mathrm{NM} x \mathrm{~A}$. Similarly fast ion densities are stored in the $2 \mathrm{D}$ variables NFAST $x(1<x<9)$ and their corresponding atomic and mass numbers are stored in 0D variables NFAST $x$ Z and NFAST $x$ A. One consequence is that impurity densities are now stored in thermal ion density variables $\mathrm{NM} x$, rendering the PR98 variable NIMP redundant. A small number of PR98 variables are redundant in PR08 (e.g. NIMP and MEFF) and have therefore been removed.

- Improvements have been made to some PR98 variable definitions (in 0D, 1D, and 2D variables) to resolve ambiguities (e.g. in PR08 the signs of toroidal quantities such as IP, CURTOT, BT now unambiguously define direction).

All PR08 variables are defined in the PR08 manual $\mathbf{~}$, and we choose not to reproduce this important but voluminous information here.

\subsection{Technology for Data Storage and Access}

PR98 data were held in fixed format ASCII files that were accessible to the public in readonly access via anonymous FTP. Four ASCII files, adhering to strict formats which are outlined in [4] II, were stored for each discharge: a comments file, a 0D data file, a 1D file and a 2D file. PR08 retains data in the form of ASCII files for backwards compatibility. The only change in file formats has been to the 0D files, and this was essential owing to the large number of additional 0D variables that were introduced in PR08. The new PR08 0D file format, which is described in the PR08 manual $\|$, is now sufficiently flexible to include only the variables that are supplied for a given discharge. This is convenient as it is rarely appropriate to supply all $1440 \mathrm{D}$ variables for any given discharge.

Data storage in the form of ASCII files has been significantly upgraded by converting the existing PR08 data to the binary tree structure files of the MDSplus system **. Developed jointly by the Massachusetts Institute of Technology, the Center for Nuclear Research (Padua, Italy) and the Los Alamos National Lab, MDSplus is the most widely used system for data management in the magnetic fusion energy programme. MDSplus stores data in a single, self-describing, hierarchical structure, and its client/server model allows data at remote sites to be read or written without file transfers and with the detailed format of the data abstracted from the user. A number of MDSplus tools are available for viewing or modifying data and its underlying structures. The MDSplus tree structure adopted for PR08 mirrors the ASCII file format of the data, and is illustrated in Figure 1(a). Below the top node in the MDSplus tree structure of each discharge there

I http://tokamak-profiledb.ukaea.org.uk/DOCS/PR08MAN/pdbman.html

||The PR98 manual is also available at http://tokamak-profiledb.ukaea.org.uk/DOCS/Manual_may_98.pdf $* *$ http://www.mdsplus.org 


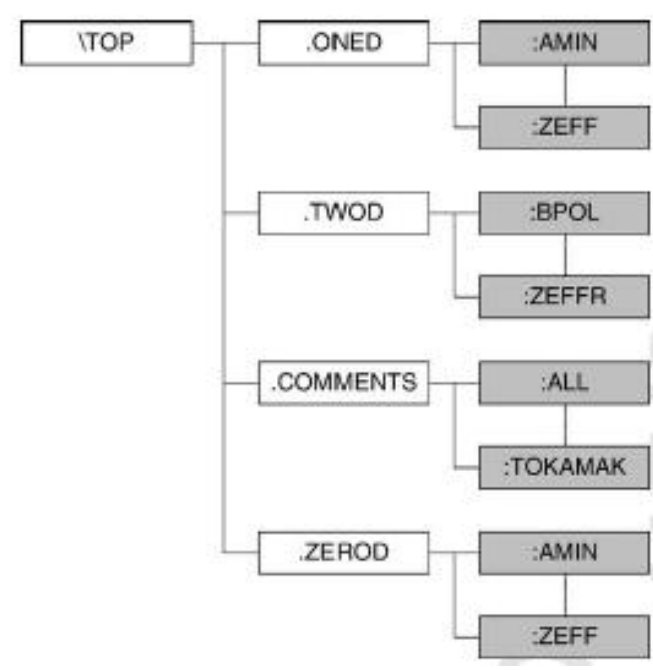

Figure 1. (a) Main branches of PR08 MDSplus tree structure are shown, along with some of the PR08 variables. (b) Main functions of the PR08 server.

are four main branch nodes, each directly relating to one of the original ASCII files, and the data variables themselves hang directly from the main branch nodes. The MDSplus tree structure is easily extendable. Once a user becomes familiar with using MDSplus for access to data, additional structure in the tree is very easily handled. (A collaboration between the ITPA CDBM and Pedestal Groups has exploited this to define an additional branch to the PR08 tree structure to describe two dimensional Grad-Shafranov equilibria, but we do not describe that here, as no two dimensional equilibria are included in PR08.) Access control to restricted data is possible with MDSplus (and we have used this), but this is not applied to PR08 as this is open to public read-only access. A conversion tool has been used to convert PR08 data from the ASCII files into MDSplus trees, and vice versa. This has been used to allow data to be submitted to the profile database in either format, and to ensure that submitted data is made available in both formats. In the future we anticipate exploiting MDSplus to extend the content of the profile database, and for MDSplus to become the predominant access method.

The infrastructure for access to PR08 has improved considerably over that for PR98, and a more technical description of these improvements has been given in [120]. http and MDSplus servers have been added to the ftp server, as illustrated in Figure 1(b), and these servers, running on a Linux system at UKAEA Culham, add considerable functionality. While the server machine is protected by a firewall, it lies outside the internal UKAEA network so that external write access can be made available to dataproviders. The http server, http://tokamak-profiledb.ukaea.org.uk, allows data to be browsed online and hosts documentation that is essential for users of the profile database: the website includes documentation on data structure and access, and hosts a number of powerful tools that are described in Section 3.3. Public and private working databases are all hosted on the machine, with appropriate access restrictions for the private databases. A further valuable improvement to the database infrastructure has been the development of a relational database to allow users to search for particular types of discharge. This relational database contains all $0 \mathrm{D}$ and comments data, and metadata for $1 \mathrm{D}$ and $2 \mathrm{D}$ variables. (The metadata consists of flags to indicate which $1 \mathrm{D}$ and $2 \mathrm{D}$ variables are 
filled, and the array sizes for these variables.) Further details of the relational database have been provided in [120]. A powerful search tool exploits this relational database, and this will be described below.

Submission of discharges to the profile database has been greatly improved by the development of a web-based submission system driven by the data-provider. Dataproviders from each tokamak have write access (via ftp or MDSplus) to their own private transit areas of the server, and web-based access to the submission system that they use to drive the transfer of their discharges from the transit area to the database itself. The submission system requires that all discharges undergo some basic (but not exhaustive) consistency checks: e.g. submitted variables must conform to the variables of PR08, and integrals of radial profile quantities should be consistent with the submitted global quantities etc. Data-providers receive feedback on the suitability of their discharges for transfer from the transit area into the database. Serious inconsistencies (such as undefined variables) prevent transfer into the database, while others are flagged as warnings for the data-provider to heed at his/her own discretion. This system has proved straightforward to use, and has helped increase the quality of submitted data by flagging common errors to the data-provider during the submission process.

\subsection{Additional Tools}

Other tools have been developed to facilitate tasks that are commonly required by users who are working with the database, and these add considerable value. One tool of

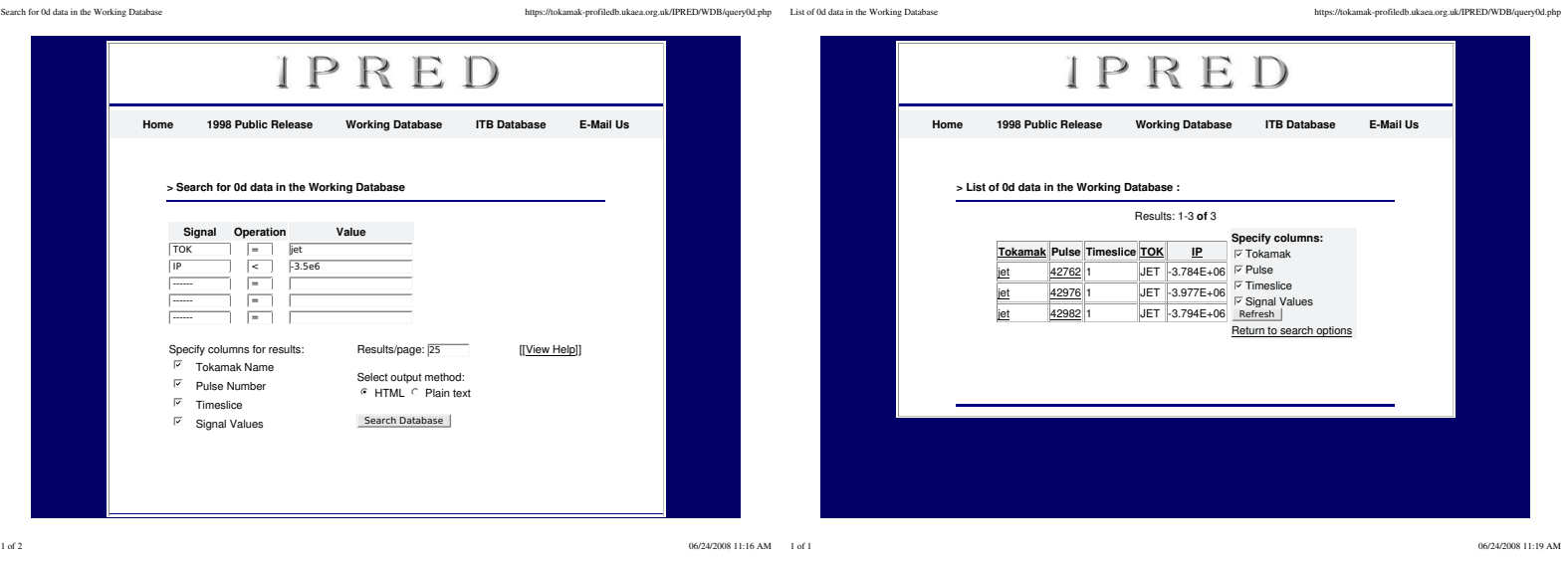

Figure 2. The lefthand plot shows a web-based IPRED search to find the highest plasma current JET discharges in PR08, while the righthand figure shows the search results. (NB, the current direction is such that IP is usually negative in JET discharges.)

interest to all users of PR08 is the IPRED search tool, which exploits the relational database to help a user find discharges of particular interest. This search tool, which can search and display 0D and comments data and assess whether variables are present, is accessible through a web interface on links from http://tokamak-profiledb.ukaea.org.uk/. An example of using IPRED to find the highest plasma current discharges in JET is illustrated in Figure 2.

Various software examples, contributed by profile database users, are available on the website to illustrate how to read profile data via MDSplus into user codes written in 
Fortran, IDL and MATLAB. Some transport codes (e.g. CRONOS [121] and JETTO [122]) can read inputs directly from the profile database to use them in transport simulations. PR08 data are sufficient, in principle, to assess whether plasma equilibria are susceptible to microinstabilities, and an IDL tool is available to generate the physics information required by the GS2 microstability code [123] $\dagger \dagger$ for a given profile database equilibrium. Linear microstability analyses of profile database discharges are also easily performed using the KineZero code $[119,124]$ ††.

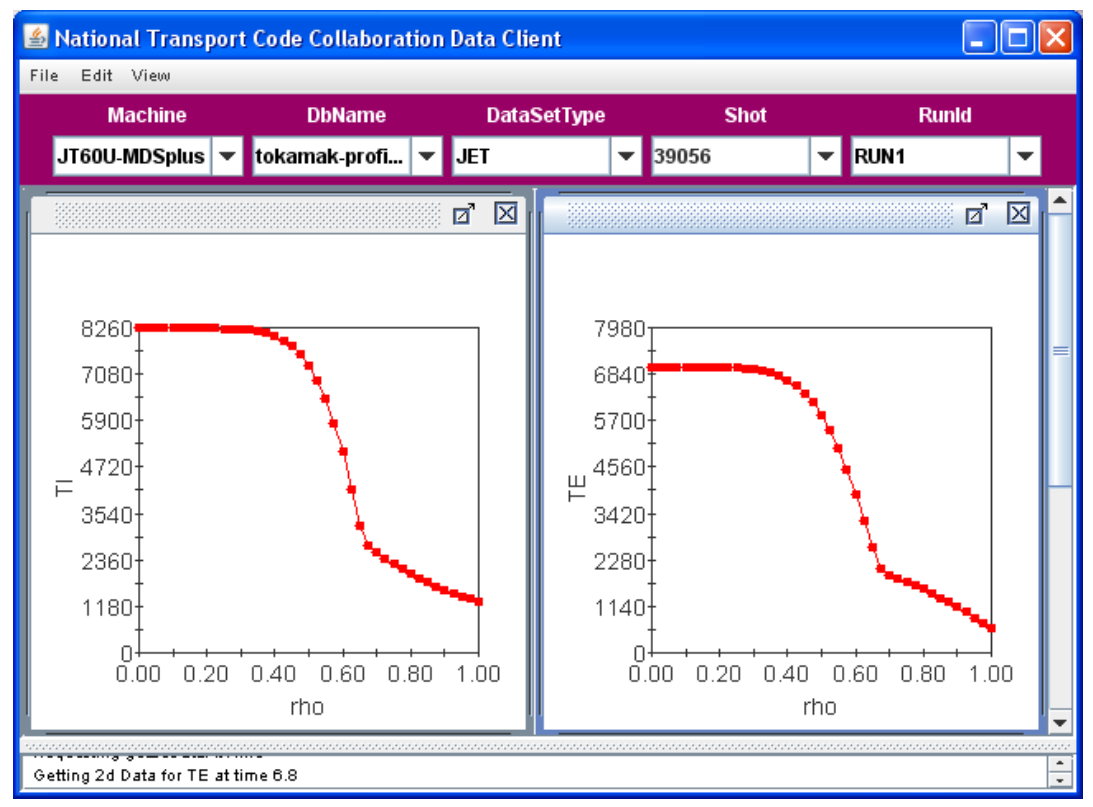

Figure 3. This ntcdata screenshot illustrates the ion (left) and electron (right) temperature profiles $(\mathrm{keV})$ as functions of the normalised flux label $\rho$ from a well developed box-like ITB in JT-60U discharge \#39056.

The ntcdata server and client [125] were developed in the US by the National Transport Code Collaboration $\S \S$. The ntcdata server reads data in multiple formats, including MDSplus trees from remote servers, and the ntcdata Java client can be accessed from a web page and provides a user friendly graphical user interface (GUI) for interactively plotting time traces and radial profiles from $1 \mathrm{D}$ and $2 \mathrm{D}$ data. A ntcdata server, accessible from the webpage http://tokamak-profiledb.ukaea.org.uk, has access to the PR08 data through MDSplus, and Figure 3 shows ion and electron temperature profiles during a well developed ITB in JT-60U to illustrate plots that can be generated using the ntcdata client.

\section{Review of Physics Analyses using PR08 Data}

Section 2 described the PR08 discharges and the most significant findings from, generally single-machine, analyses of the data. The profile database facilitates more powerful multimachine tests of models over broader ranges of parameters, and here we provide an

††http://gs2.sourceforge.gs2.net

†thttp://tokamak-profiledb.ukaea.org.uk/KZERO/KINEZERO.htm

$\S \S$ http://w3.pppl.gov/ntcc 
overview of such modelling activity that has made use of the profile database, referring the interested reader to the cited literature for details. The testing of core transport models in L-mode and H-mode discharges was the main thrust of physics activity using PR98 and has been described in $[1,4,5]$ : one important emphasis of that effort was the development of rigorous protocols for the testing of models by multiple authors in standardised codes. This topic remains of considerable importance and interest, but since PR98 the modelling community has diversified to consider further pertinent and important phenomena, such as ITBs.

Transport model developers have continued to make increasingly detailed comparisons with experimental data from L-mode and H-mode discharges, including assessing how the quality of model predictions varies with parameters like $\rho_{*}$ and $T_{e} / T_{i}$. The rigorous protocols for the testing of models by multiple authors, developed in $[1,4,5]$, were most closely followed in [126]: (i) the transport code developed was successfully tested both against a simple analytic benchmark case and against results from an independent code for the more complex transport models of interest; and (ii) the statistical figures of merit proposed in [1] were used to quantify the consistency of simulations with experimental data. Simulations using the current diffusive ballooning mode (CDBM) [127], Weiland [128], and GLF23 [129] transport models were compared with experimental data from 54 L-mode and H-mode discharges from DIII-D, JET, JT$60 \mathrm{U}$ and TFTR (taken from the profile database) [126]. Averaged over all discharges, the GLF23 model predictions had the lowest mean RMS deviation in the incremental stored energy. H-mode and L-mode discharges were found to be most accurately simulated using the GLF23 and CDBM models respectively. Enhancements to the CDBM model to describe elongated plasmas were found to improve the predictions [130].

Few other works in this area have been able to adopt the rigorous protocols of references $[1,4,5]$ so fully, though many have been influenced by that work and have used the figures of merit that were proposed. The mixed Bohm/gyro-Bohm [131] and Multi-mode (MMM95) [132] models have been compared with 13 L-mode and 22 H-mode discharges from JET, DIII-D and TFTR in references [133] and [134] respectively. These discharges were taken from the profile database, and included systematic scans in $\rho_{*}, \beta$, collisionality, isotope mass, $\kappa$, power, current and density. The predicted profiles from both models were found to match the data equally well, giving root mean square (RMS) deviations between the modelled and experimental ion temperature profiles close to $10 \%$. The mixed Bohm/gyro-Bohm model was generally dominated by Bohm scaling transport over most of the plasma cross section, while the MMM95 modelled transport had a pure gyro-Bohm scaling. Changes in profile shapes through the $\rho_{*}$ scans allowed both models to remain consistent with the experimentally observed changes in global confinement. The canonical profiles transport model (CPTM) has been tested by comparing simulation results with experimental measurements from 45 discharges from eight different tokamaks (DIII-D, JET, JT-60U, MAST, TFTR, ASDEX-U, T10 and TEXTOR), 32 of which were taken the profile database [135]. The CPTM predictions of the electron temperature gradient parameter $\Omega_{T_{e}}=-\left(R / T_{e}\right) d T_{e} / d r$ were found to agree with the measured values to within $\sim 15 \%$ over a wide range of $\Omega_{T_{e}}\left(2<\Omega_{T_{e}}<12\right)$.

In most transport models, anomalous transport is triggered when a critical value 
of $R / L_{T}$ is exceeded, and modelled fluxes in the core are sensitive to the properties of the edge pedestal. Fully predictive simulations for new devices clearly require an integrated approach where the core transport and pedestal properties are included in the model. The MMM95 core transport model was integrated with a pedestal model, and simulations have been compared with 33 JET and DIII-D H-mode discharges from the profile database [136]. The average RMS deviations between the predicted and experimental profiles of temperature and density, normalised to the central values, was close to $10 \%$.

Sawtooth oscillations affect confinement in tokamaks. A sawtooth model, consisting of the triggering mechanism proposed by Porcelli [137] and Kadomtsev reconnection [138], was compared with data from 17 TFTR and JET discharges from the profile database [139]. The mechanisms responsible for triggering the crashes were investigated, and it was found that the modelled sawtooth periods best matched those observed when the model's reconnection fraction parameter was set to $37 \%$.

Particle transport was investigated in [140], using a modified version [141] of the BALDUR code [142] , for 34 profiles from ASDEX Upgrade, 8 H-mode profiles from JET and DIII-D (from the profile database) and 4 profiles from ASDEX. Statistical analysis of the ASDEX Upgrade data alone was used to derive a two-term scaling for the normalised particle pinch, $r v_{\text {in }} / D$ (where $r$ is the plasma minor radius, $v_{\text {in }}$ is the particle pinch velocity and $D$ is the anomalous particle diffusivity). The scaling comprised of a 'principal' term depending on $-\left(a / T_{e}\right)\left(d T_{e} / d r\right)$, and a secondary term depending on loop voltage and collisionality. The scaling derived from ASDEX Upgrade data described the other devices well, increasing confidence that the model could provide reliable predictions of particle confinement in ITER.

Internal transport barriers considerably enhance confinement, and improving our understanding could assist the achievement of higher performance burning plasmas in next step devices. Studies of the physics underlying ITBs via international multi-machine comparisons have been reviewed in [143] and more recently in [2], and have been facilitated using the ITB profile database [144], which is included in PR08. Gohil et al drew two important findings from analyses of ITB discharges from global and profile databases gathered from a wide range of machines [144]. Firstly the threshold input power $P_{\text {th }}$ required to generate an ITB was reduced with negative magnetic shear. Secondly, in ITBs the growth rates of microinstabilities lay close to the radial shear of equilibrium flow $\omega_{\text {ExB }}$, which suppressed the mechanisms that drive turbulent fluctuations. Gyrokinetic calculations found reduced growth rates at negative magnetic shear, consistent with the reduced power thresholds observed at negative magnetic shear. High pressure gradients in ITB plasmas can be expressed in terms of the normalised pressure gradient parameter $\alpha$. High $\alpha$ reduces the magnetic drifts, and gyrokinetic calculations have demonstrated that this can stabilise microturbulence. Comparisons with data from ITB discharges in the profile database have confirmed that $\alpha$ stabilisation is indeed important in experimental plasmas with ITBs across machines [124]. Transport models have been modified over recent years to improve their capability to describe plasmas with ITBs. The GLF23 model, for example, which already includes the important ingredients of $\alpha$ stabilisation and sheared equilibrium flow, was 'retuned' in 2005 to improve its approximations for 
linear microinstability growth rates in reversed magnetic shear plasmas and in the Hmode pedestal [145]. With these improvements, that model improved its capability to describe steady plasmas with ITBs [145]. Improvements to the mixed Bohm/gyro-Bohm model, to include $\alpha$ stabilisation and equilibrium flow shear, were described in [146]. Predictive modelling of time-dependent ITB plasmas is considerably more demanding than simulating steady ITBs. A recent use of the profile database has been to attempt such time-dependent calculations for 2 ITB discharges from each of DIII-D, JET and JT-60U using the mixed Bohm/gyro-Bohm, GLF23 and Weiland transport models [146]. The Bohm/gyro-Bohm model simulated from the preheating phase through to the high performance phase in all 6 discharges with fair accuracy: the $\alpha$ stabilisation term in this model was essential for the desciption of the DIII-D discharges. The Weiland model failed to reproduce ITBs in these simulations, although the average temperatures and densities were close to those in the experiment. The GLF23 model predicted ITBs, but usually at lower minor radius than in experiment and therefore tended to underestimate the plasma performance.

Achieving steady state operation is an important goal for future burning plasma devices, and longer pulse scenarios are being sought by exploiting non-inductive current sources and the self-driven bootstrap current. Advanced tokamak and hybrid scenarios have recently been developed on a number of machines in order to work towards this goal. The essential difference between AT and hybrid scenarios is that the core safety factor is close to 1 in the hybrid scenario, and larger in the AT scenario. The plasma current is reduced in both modes (so reducing confinement), but it has been found experimentally that with careful tuning of the plasma current profile the energy confinement can be optimised. Considerable effort has been invested in trying to understand the influences on plasma confinement in these regimes. A number of hybrid and AT discharges from a range of machines are included in PR08 and are accessible to modellers for detailed transport modelling and microstability analysis. Microstability studies and the GLF23 transport model were used to demonstrate that equilibrium ExB flow shear and $\alpha$ stabilisation played crucial roles in improving confinement in AT and hybrid plasmas from DIII-D, JET and ASDEX Upgrade [147]: $\alpha$ and flow shear stabilisation were more important in AT and hybrid plasmas respectively. The profile database facilitated transport modelling and microstability calculations for a single time slice from 7 hybrid discharges from ASDEX Upgrade, DIII-D, JET and JT-60U [59]. The simulations presented using the Weiland and GLF23 transport models showed strong and unpredictable inter-shot variations in the quality of their agreement with the experimental data. One puzzle posed in [59] was that some discharges were found to be stable to all microinstabilities in the region $\rho<0.6$, whereas the experimentally observed transport (and that predicted by the Weiland and GLF23 models) was anomalous in this region. Moreover the inter-shot variation of the confinement enhancement factors, over the H-mode confinement scaling law $\tau_{\text {IPB98,y2 }}[1]$, did not correlate with changes in either the GLF23 or the Weiland model descriptions of the core transport processes. 


\section{Summary and Conclusions}

The PR08 public release of the international multi-tokamak confinement profile database is a significant extension of the 1998 public release of the ITER profile database, PR98 [4]. PR08 supplements PR98 with approximately one hundred new experimental discharges including: world record fusion power discharges from DT plasma operation in JET and TFTR; discharges with internal transport barriers, from JET, JT-60U and DIII-D; high performance hybrid scenario discharges from DIII-D and JT-60U; H-mode parameter scans from AUG; low aspect ratio plasmas from the spherical tokamak MAST; and electron heated discharges from FTU, T-10 and Tore Supra. Simulations of a set of possible ITER scenarios (described in Appendix A) are also included in PR08.

These data, which are of considerable interest to the wider magnetic confinement fusion community, are now openly available read-only through ftp, http and MDSplus. A variety of powerful tools are also available to facilitate many of the most common tasks undertaken by users working with the data. All tools, data structures and access methods are well documented on an extensive website: http://tokamak-profiledb.ukaea.org.uk. While public access to PR08 data is unconditional, this paper should be cited in any publication that makes use of PR08 data.

\section{Acknowledgments}

We are grateful to J W Connor and J G Cordey who have been strong supporters of the profile database, and acknowledge substantial assistance on IT issues from $\mathrm{N}$ Cook, O Hemming, P J Knight, R Layne, S Rahman, D Robson, M Brooks and J Waterhouse at UKAEA. We are also grateful to M F Turner for explaining how the profile database had been set up prior to its move to UKAEA in 2001. This work was supported in part by the US Department of Energy under DE-AC02-76CH03073 (PPPL), DE-FC02-04ER54698 (GA), DE-AC05-00OR22725 (ORNL), DE-FC02-99ER54512 and DE-FG02-07ER54917, by the United Kingdom Engineering and Physical Sciences Research Council, and by

the European Communities under the contract of Association between EURATOM and UKAEA. The views and opinions expressed herein do not necessarily reflect those of the European Commission. 


\section{Appendix A. Transport Simulations of Possible ITER Scenarios}

The main reference scenarios that are envisaged for ITER have been defined in [148-150]: the fully inductive reference scenario has plasma current $I_{p}=15 \mathrm{MA}, Q \geq 10$, current flattop duration $\Delta t=300-500 \mathrm{~s}$ and neutron flux to the wall $F_{N} \geq 0.5 \mathrm{MWm}^{-2}$; the hybrid reference scenario has partial non-inductive current drive with $Q>5$ and current flattop duration $\Delta t>1000 \mathrm{~s}$; and the steady state reference scenario has fully non-inductive current drive with $Q>5$ and current flat-top duration $\Delta t>3000$ s. PR08 includes simulations of ITER inductive, hybrid and steady state (or advanced tokamak) scenarios, and these are tabulated in Table A1.

\begin{tabular}{|c|c|c|c|l|}
\hline Shot number & $\begin{array}{c}I_{p} \\
(\mathrm{MA})\end{array}$ & $\begin{array}{c}P_{\text {aux }} \\
(\mathrm{MW})\end{array}$ & $\begin{array}{c}P_{\text {fus }} \\
(\mathrm{MW})\end{array}$ & $\begin{array}{l}\text { Transport } \\
\text { Code }\end{array}$ \\
\hline 10010100 & 15 & 33 & 411 & PTRANSP \\
10020100 & 14.72 & 53 & 518 & PTRANSP \\
20010100 & 12.08 & 33 & 332 & PTRANSP \\
20020100 & 12.03 & 53 & 598 & PTRANSP \\
20030100 & 12.05 & 33 & 546 & PTRANSP \\
20040100 & 12.1 & 53 & 530 & PTRANSP \\
10020201 & 15 & 40 & 408 & ASTRA \\
10020202 & 15 & 33 & 441 & ASTRA \\
10020203 & 15 & 33 & 384 & ASTRA \\
10030201 & 15 & 73 & 402 & ASTRA \\
10050201 & 17 & 35 & 705 & ASTRA \\
30040201 & 9 & 68 & 339 & ASTRA \\
30040202 & 9 & 73 & 347 & ASTRA \\
20120300 & 11.3 & 53 & 280 & CRONOS \\
20140300 & 11.3 & 53 & 381 & CRONOS \\
20310300 & 13 & 53 & 579 & CRONOS \\
20350300 & 13 & 53 & 723 & CRONOS \\
20370300 & 13 & 53 & 849 & CRONOS \\
20380300 & 13 & 53 & 680 & CRONOS \\
20390300 & 13 & 53 & 223 & CRONOS \\
20410300 & 13 & 53 & 268 & CRONOS \\
\hline
\end{tabular}

Table A1. Some key parameters from the ITER scenario simulations that are included in PR08. The shot numbers are of the form: "sdddmmrr", where $s$ flags the scenario ( $s=$ 1 for Elmy H-mode, 2 for hybrid and 3 for steady state or advanced tokamak), $d d d$ is a discharge label within a specific scenario, $m m$ denotes the modeller (01=Robert Budny, $02=$ Alexei Polevoi, $03=$ Frederic Imbeaux), and $r r$ is a run number index starting from zero $(00,01,02, \ldots, 98,99)$.

\section{Appendix A.1. PTRANSP Simulations of ELMy H-mode and Hybrid Scenarios}

The first six discharges in Table A1 are from time-dependent integrated modelling simulations using the PTRANSP code [151]. In the first simulated discharge (\#10010100) the electron density, and ion and electron temperature profiles were taken from [152] in an interpretive transport analysis to determine self-consistent transport coefficients. The other five simulated discharge were modelled in two steps. In the first step the Tokamak 
Simulation Code, TSC $[153,154]$, was used to model self-consistent plasma equilibrium and tranport evolution (with prescribed evolution of the density profile) from an early limiter phase through to the current flat-top. The GLF23 transport model [129] was used in TSC, with the default settings from the NTCC Module Library [155] and including turbulence stabilisation terms from $\alpha$ and equilibrium flow shear. In a second step the TSC profiles were fed into PTRANSP for more detailed analysis of heating, current drive, sources of torque and fuelling $*$. PTRANSP used the shaped plasma boundary evolution from TSC, and calculated all other plasma parameters self-consistently. Equilibria (with no assumption of up/down symmetry) were calculated in PTRANSP using ESC [107] and VMEC [106]. Predictions of plasma performance were sensitive to the boundary temperatures prescribed near the top of the edge pedestal, and which have been taken, consistent with pedestal scalings [156], as $5.6 \mathrm{keV}$ for the H-mode plasmas. Auxiliary heating power in reference high performance ITER plasmas was assumed to comprise: between 16.5MW and 33MW of D-NNBI (using one or two beam lines of negative ion neutral beam injection, with $\mathrm{D}$ injection energy of $1 \mathrm{MeV}$ ) and up to $20 \mathrm{MW}$ of ICRH, with frequency tuned to the $\mathrm{He}^{3}$ minority resonance near the plasma center $(\sim 53 \mathrm{MHz})$. PTRANSP uses the Monte Carlo code NUBEAM [157] to model alpha particle heating, neutral beam heating, fuelling, torque, and current drive, and can model situations where the NNBI plasma footprint is displaced vertically from the magnetic axis. ICRH modelling assumed a coupling to $\mathrm{He}^{3}$ minority ions (the assumed $\mathrm{He}^{3}$ density $n_{H e}$ is $n_{H e} / n_{e}=2-3 \%$ ) and uses the SPRUCE module [72]. Impurities were assumed to consist of beryllium and argon: $n_{B e} / n_{e}=2 \%$ and $n_{A r} / n_{e}=0.12 \%$. Helium ash accumulation was modelled by computing the alpha particle thermalisation rate and with assumed values for the particle diffusivity, pinch velocity, and recycling coefficient for He. Plasma rotation was modelled by assuming a fixed value of the ratio of the angular momentum to ion energy effective diffusivities, $\chi_{\phi} / \chi_{i}$. Sawtooth mixing of current and fast ions was modelled using a modification of the Kadomtsev model [138], and this significantly affected the $q$-profiles and the distributions of fast ions (both beam ions and fusion alpha particles).

The first two plasmas in Table A1 (\#10010100 and \#10020100) are H-mode simulations, where the electron density profile was assumed to be flat and to ramp up to a steady state Greenwald fraction of 0.8 . The normalised pressure parameter, $\beta_{N}$, was 1.80: sufficiently low that neoclassical tearing modes should not be expected to degrade energy confinement.

The next four plasmas in Table A1 (\#20010100, \#20020100, \#20030100, \#20040100) are hybrid discharges with lower $I_{p}$, higher bootstrap current and higher $\beta_{N}$ (ranging from 2.1 to 3.1). Higher pedestal temperatures $\simeq 10 \mathrm{keV}$ were assumed in the hybrid simulations, but modelled temperature profiles were similar to those in H-mode. One key characteristic of hybrid plasmas is that the central $q$-profile remains close to or slightly above one, which helps avoid confinement degradation due to neoclassical tearing modes. Large sawteeth would be expected if $q_{\text {min }}$ were to drop sufficiently below one, but PTRANSP simulations suggest that below-axis NNBI can delay this for at least 500s [57].

Density profile peaking on ITER is uncertain, but experiments have found that

*PTRANSP results could be fed back into TSC for further iteration, but this was not done for the simulations in PR08. 
density profiles become more peaked as the normalised collisionality $\nu_{*}$ reduces towards the ITER value [158]. In two of the hybrid ITER plasmas where peaked density profiles were assumed (\#20030100 and \#20040100), PTRANSP simulations with the GLF23 model predicted reduced fusion power due to increased transport from trapped particle modes.

\section{Appendix A.2. ASTRA Simulations of ELMy H-mode and Hybrid Scenarios}

Transport models, with confinement times guided by the H-mode scaling law $\tau_{\text {IPB98,y2 }}$ [1], were used for the assessment of plasma performance in the inductive ITER baseline scenario (with $\beta_{N}<2.5, n / n_{G}<1, q_{95} \sim 3$, and with impurity concentrations consistent with $Z_{\text {eff }}<2$ ) [159-161]. In these simulations, transport coefficients were scaled to enhance the modelled energy confinement time by a prescribed factor $H_{98 y 2}$ over the scaling law, such that $\tau_{E}=H_{98 y 2} \tau_{\text {IPB98,y2 }}$. Radial transport equations for temperatures of electrons and ions, densities of electrons and helium, toroidal rotation and poloidal magnetic flux, were solved in the plasma core and pedestal regions. Core transport coefficients were scaled by a constant factor to ensure $H_{98 y 2}=1$ for the inductive scenario, and to achieve $Q=5$ in long pulse operation. The H-mode pedestal width was taken from the model of reference [162]. Electron source $S_{e}$ contributions from edge gas puffing, pellet and NBI were computed. The diffusion equation for poloidal magnetic flux used neoclassical current conductivity [10], and included the sources from bootstrap current $j_{\text {bs }}[163]$ and externally driven currents $j_{\mathrm{CD}}$. When $q_{0}$ fell below 1 sawteeth were modelled by flattening plasma temperature, density and current profiles in the region $r<1.4 r_{q=1}$. Simulations with this simple integrated model of transport and sawteeth demonstrated reasonable agreement with experimental data from improved confinement regimes [161], and with predictions for ITER scenarios using more sophisticated transport models [164]. Helium source contributions were calculated from fusion reactions and from ionisation of the He influx from the boundary. Densities of other impurity species were taken as fixed fractions of the electron density. The beryllium concentration was taken as $n_{\mathrm{Be}} / n_{e}=0.02$. Argon impurities were prescribed to provide safe divertor operation with an acceptable power loss through the separatrix $\left(P_{\text {loss }}<110 \mathrm{MW}\right)$, and to moderate the peaking of plasma pressure to give stable operation below the ideal wall limit in long pulses with an ITB [160]. Effective impurity charges were determined using the coronal equilibrium model [165], and fuel densities $n_{D}$ and $n_{T}$ were forced to be consistent with quasineutrality. Modelling of long pulse and steady state scenarios used the transport model described, with the following additional ingredient to describe ITBs: in low or reversed magnetic shear regions all transport coefficients were reduced to the neoclassical ion heat diffusivity [1,159]. ASTRA [9] was used with the above transport models to assess various ITER scenarios.

Simulations of ITER inductive reference scenarios 2, 3 and 5 from [150], first carried out using the PRETOR code [166], were reproduced using ASTRA [167] and included in PR08 as ITER discharges \#10020201, \#10030201 and \#10050201 respectively. Relatively high values for the pedestal density $\left(6 \times 10^{19} \mathrm{~m}^{-3}\right)$ and edge temperature $(1 \mathrm{keV})$ were assumed, and the argon impurity fraction $n_{\mathrm{Ar}} / n_{e}$ was taken as $0.12 \%$. In ASTRA NBI was calculated using a single off-axis pencil beam $P_{\mathrm{NBI}}=33 \mathrm{MW}$, and central RF heating was simulated using a parabolic power density profile with heating divided equally between 
ions and electrons.

Recent progress in integrated modelling has improved on the original PRETOR ITER baseline scenario simulations. Boundary conditions at the separatrix are calculated selfconsistently by using the B2-Eirene code [168] to compute heat and particle transport in the scrape-off-layer and divertor. Integrating such simulations with core transport modelling allows neutral fuelling, impurity transport, core density and temperature profile evolution, and the losses of heat and particles to be calculated self-consistently [169]. Such calculations predict that: core fuelling from edge neutrals saturates at a low level $S_{\text {DT,core }}<10^{22}$ atoms $/ \mathrm{s}, n_{e}^{\text {ped }} \sim 3-4 \times 10^{19} \mathrm{~m}^{-3}, T_{e}^{\text {ped }} \sim 200 \mathrm{eV}$, the He atomic influx is $50 \%$ of the outflux, fusion power $P_{\text {fus }}=400-500 \mathrm{MW}$, and $P_{\text {loss }}<100-110 \mathrm{MW}$. Inductive baseline reference scenarios were calculated with ASTRA, using B2-Eirene data for edge fuelling and with sufficient pellet core fuelling to reach the design density [170]. These are included in PR08 discharges \#10020202 and \#10020203.

PR08 discharges \#20040201 [160] and \#20040202 [171] are ASTRA simulations of the long pulse reference scenario 4 from [150], using: boundary conditions from B2-Eirene; helium transport; and realistic NBI with $\left(P_{\mathrm{NBI}}=34 \mathrm{MW}\right)$. In $\# 20040201\left(f_{N I}=1\right)$ LHCD was prescribed with a simple model, while in \#20040202 $\left(f_{N I}=0.955\right)$ ECCD and LHCD were calculated with the OGRAY [172] and FRTC [173] codes respectively. These latter models demonstrated good agreement with more sophisticated ITER current drive simulations $[174,175]$.

\section{Appendix A.3. CRONOS Simulations of Hybrid Scenarios}

The simulations carried out with the CRONOS code [121], were for the hybrid scenarios that were described in [59]. These simulations were performed with two different values of the plasma current $\left(I_{p}=11.3 \mathrm{MA}\right.$ and $\left.I_{p}=13 \mathrm{MA}\right)$, and various transport models were assumed [59]: in \#20120300, \#20310300, \#20350300, \#20390300, \#20410300, \#20140300 and \#20370300 the assumed thermal diffusivities had a radial profile shape

$\propto q^{2} \sqrt{T}|\nabla p| / n_{e}$ and were normalised to fit scaling expressions for the global confinement, \#20380300 used the GLF23 model in the region $0.3<\rho<0.8$. Simulations \#20140300 and \#20370300 demonstrated the impact of off-axis Lower Hybrid current drive, where the appearance of the $q=1$ surface in the plasma was significantly delayed. Further details on these simulations are available in [59].

\section{References}

[1] ITER Physics Expert Groups on Confinement and Transport and Confinement Modelling and Database, et al, Nuclear Fusion 39, 2175 (1999).

[2] E. J. Doyle et al., Nuclear Fusion 47, S18 (2007).

[3] J. W. Connor et al, Validation of 1-D Transport and Sawtooth Models for ITER, in Fusion Energy 1996 (Proc. 16th Int. Conf. Montreal 1996), Vol. 2, p. 935, IAEA, Vienna, (1997).

[4] The ITER 1D Modelling Working Group: D Boucher et al., Nuclear Fusion 40, 1955 (2000).

[5] D. R. Mikkelsen et al., Tests of Tokamak Transport Models, in Proceedings of 26th EPS Conference on Controlled Fusion and Plasma Physics, Maastricht, Vol. ECA Vol 23J, p. 1677, 1999.

[6] A. Herrmann and O. Gruber, Fusion Science and Technology 44, 569 (2003).

[7] G. Tardini et al., Nuclear Fusion 42, 258 (2002).

[8] P. J. McCarthy and F. C. Morabito, Int. J. of App. Electrom. and Mechanics 9, 1 (1997). 
[9] G. V. Pereverzev and P. N. Yushmanov, IPP report 5/98 and http://www.ipp.mpg.de/ippcms/de/kontakt/bibliothek/ipp_reports/IPP_5_98.pdf (2002).

[10] S. P. Hirshman, R. JHawryluk, and B. Birge, 17, 611 (1977).

[11] J. L. Luxon et al., in Plasma Physics and Controlled Nuclear Fusion Research 1986 (Proc. 11th Int. Conf., Kyoto, 1986), Vol. 1, p. 159, IAEA, Vienna, (1987).

[12] J. L. Luxon et al., Fusion Science and Technology 48, 807 (2005).

[13] R. J. Goldston et al., J Comput Phys 43, 61 (1981).

[14] L. L. Lao et al., Nuclear Fusion 25, 1611 (1985).

[15] A. Pankin et al., Computer Physics Communications 159, 157 (2004).

[16] C. C. Petty et al., Physics of Plasmas 9, 128 (2002).

[17] P. N. Yushmanov et al., Nuclear Fusion 30, 1999 (1990).

[18] M. Murakami et al., Nuclear Fusion 40, 1257 (2000).

[19] G. R. McKee et al., Physics of Plasmas 7, 1870 (2000).

[20] M. Murakami et al., Nuclear Fusion 41, 317 (2001).

[21] C. M. Greenfield et al., Physics of Plasmas 4, 1596 (1997).

[22] K. H. Burrell et al., Plasma Physics and Controlled Fusion 40, 1585 (1998).

[23] E. J. Doyle et al., Plasma Physics and Controlled Fusion 42, A237 (2000).

[24] B. W. Stallard et al., Physics of Plasmas 6, 1978 (1999).

[25] C. Greenfield et al., Improvement of Core Barriers with ECH and Counter-NBI in DIII-D, in Proceedings of 27th EPS Conference on Plasma Physics and Controlled Fusion, Budapest, Hungary, Vol. ECA 24B, P2-022, p. 544, 2000.

[26] E. J. Doyle et al., Nuclear Fusion 42, 333 (2002).

[27] C. M. Greenfield et al., Physical Review Letters 86, 4544 (2001).

[28] K. H. Burrell et al., Physics of Plasmas 8, 2153 (2001).

[29] E. J. Doyle et al., Plasma Physics and Controlled Fusion 43, A95 (2001).

[30] C. M. Greenfield et al., Physics of Plasmas 7, 1959 (2000).

[31] M. R. Wade et al., Physics of Plasmas 8, 2208 (2001).

[32] M. Murakami et al., Physical Review Letters 90, 255001 (2003).

[33] M. Murakami et al., Physics of Plasmas 10, 1691 (2003).

[34] M. R. Wade et al., Nuclear Fusion 43, 634 (2003).

[35] M. R. Wade et al., Self-Consistent Integrated Advanced Tokamak Operation in DIII-D, in Fusion Energy 2002 (Proc. 19th Int. Conf. Lyon, 2002) (Vienna: IAEA) CD-ROM file PD/T-1 and http://www.iaea.org/programmes/ripc/physics/fec2002/html/fec2002.htm.

[36] T. C. Luce et al., Nuclear Fusion 41, 1585 (2001).

[37] M. R. Wade et al., Nuclear Fusion 45, 407 (2005).

[38] R. Andreani et al., in Proceedings of 16th Symposium on Fusion Technology London, 1990, Vol. 1, p. 2118, 1991.

[39] D. Frigione et al., Nuclear Fusion 41, 1613 (2001).

[40] B. Esposito et al., Plasma Physics and Controlled Fusion 46, 1793 (2004).

[41] M. Romanelli et al., Nuclear Fusion 46, 412 (2006).

[42] G. Bracco et al., ECRH Results during Current Ramp-up and Post-Pellet Injection in FTU Plasma, in Fusion Energy 2000 (Proc. 18th Int. Conf. Sorrento, 2000) (Vienna: IAEA) CD-ROM file EX6/3 and http://www.iaea.org/programmes/ripc/physics/fec2000/html/node1.htm.

[43] G. Saibene et al., Plasma Physics and Controlled Fusion 44, 1769 (2002).

[44] J. Ongena et al., Plasma Physics and Controlled Fusion 43, A11 (2001).

[45] P. Dumortier et al., Plasma Physics and Controlled Fusion 44, 1845 (2002).

[46] M. Valovic et al., Plasma Physics and Controlled Fusion 44, 1911 (2002).

[47] J. Ongena et al., Nuclear Fusion 44, 124 (2004).

[48] W. Suttrop et al., in Proceedings of 28th EPS Conference on Plasma Physics, Madeira, Vol. ECA 25A, P3-016, p. 989, 2001.

[49] L. D. Horton et al., Nuclear Fusion 39, 993 (1999).

[50] M. Keilhacker et al., Nuclear Fusion 39, 209 (1999).

[51] R. V. Budny, Nuclear Fusion 42, 1383 (2002).

[52] G. Bateman et al., Phys Plasmas 6, 4607 (1999). 
[53] R. V. Budny et al., Phys Plasmas 7, 5038 (2000).

[54] R. V. Budny et al., Nuclear Fusion 42, 66 (2002).

[55] R. Sartori et al., in Proceedings of 28th EPS Conference on Plasma Physics, Madeira, Vol. ECA 25A, P3-003, p. 937, 2001.

[56] I. Voitsekhovitch et al., in Proceedings of 33rd EPS Conference on Plasma Physics, Rome, Vol. ECA 30I, pp. P1-078, 2006.

[57] R. V. Budny et al., Transport Physics of Hybrid Scenario Plasmas in the International Multi-Tokamak Database and Implications for ITER, in Fusion Energy 2006 (Proc. 21st Int. Conf. Chengdu, 2006) (Vienna: IAEA) CD-ROM file IT/P1-5 and http://wwwnaweb.iaea.org/napc/physics/FEC/FEC2006/html/index.htm, pp. (IT/P1-5), 2006.

[58] E. Joffrin et al., Nuclear Fusion 45, 626 (2005).

[59] F. Imbeaux et al., Plasma Physics and Controlled Fusion`47, B179 (2005).

[60] F. X. Söldner et al., Nuclear Fusion 39, 407 (1999).

[61] F. X. Söldner and the JET Team, Plasma Physics and Controlled Fusion 39, B353 (1999).

[62] JET Team (prepared by A C C Sips), Nuclear Fusion 41, 1559 (2001).

[63] I. Voitsekhovitch et al., Phys Plasmas 6, 4229 (1999).

[64] I. Voitsekhovitch et al., Czechoslovak Journal of Physics 49 Suppl. S3, 41 (1999).

[65] E. Joffrin et al., Nuclear Fusion 42, 235 (2002).

[66] C. D. Challis et al., Plasma Physics and Controlled Fusion 44, 1031 (2002).

[67] Y. Baranov et al., Plasma Physics and Controlled Fusion 46, 1181 (2004).

[68] R. V. Budny et al., Plasma Physics and Controlled Fusion 44, 1215 (2002).

[69] X. Litaudon et al., Plasma Physics and Controlled Fusion 44, 1057 (2002).

[70] R. C. Wolf et al., Plasma Physics and Controlled Fusion 45, 1757 (2003).

[71] G. M. D. Hogeweij et al., Plasma Physics and Controlled Fusion 44, 1155 (2002).

[72] M. Evrard, J. Ongena, and D. van Eester, Improved Dielectric Tensor in the ICRH module of TRANSP, in AIP Conference Proceedings: 11th Topical Conference on Radio Frequency Power in Plasmas, 11th topical conference, Palm Springs, Vol. 335, p. 235, 1995.

[73] W. A. Houlberg, K. C. Shaing, and S. P. Hirshman, Physics of Plasmas 4, 3230 (1997).

[74] J. Weiland, Collective modes in inhomogeneous plasma. Kinetic and advanced Fluid theory Plasma Physics Theory, Institute of Physics Publishing (Bristol), 2000.

[75] A. L. Rogister, Phys Plasmas 7, 5070 (2000).

[76] X. Garbet et al, Phys Plasmas 8, 2793 (2001).

[77] H. Takenaga and the JT-60 Team, Nuclear Fusion 47, S563 (2007).

[78] S. Ide and the JT-60 Team, Nuclear Fusion 45, S48 (2005).

[79] H. Kishimoto et al., Nuclear Fusion 45, 986 (2005).

[80] H. Shirai et al., Plasma Physics and Controlled Fusion 42, 1193 (2000).

[81] N. Hayashi et al., Nuclear Fusion 47, 682 (2007).

[82] T. Fujita et al., Fusion Engineering Design 34-35, 289 (1997).

[83] K. Tani et al., Journal of the Physical Society of Japan 50, 1726 (1981).

[84] K. Hamamatsu and A. Fukuyama, Fusion Engineering Design 53, 53 (2001).

[85] S. V. Neudatchin et al., in Fusion Energy 2000 (Proc. 18th Int. Conf. Sorrento, 2000) (Vienna: IAEA) CD-ROM file EXP5/01 and http://www.iaea.org/programmes/ripc/physics/fec2000/html/node1.htm.

[86] S. V. Neudatchin et al., Journal of Plasma Fusion Research 79, 1218 (2003).

[87] A. Isayama et al., Nuclear Fusion 43, 1272 (2003).

[88] A. Isayama et al., Physics of Plasmas 12, 056117 (2005).

[89] H. Takenaga et al., Nuclear Fusion 43, 1235 (2003).

[90] R. J. Akers et al., Plasma Physics and Controlled Fusion 45, A175 (2003).

[91] A. R. Field et al., Core Heat Transport in the MAST Spherical Tokamak, in Fusion Energy 2004 (Proc. Int. Conf. Vilamoura, 2004) (Vienna: IAEA) CD-ROM file EX/P2-11 and http://wwwnaweb.iaea.org/napc/physics/fec/fec2004/datasets/index.html.

[92] C. M. Roach et al., Plasma Phys Control Fusion 47, B323 (2005).

[93] D. J. Applegate et al., Plasma Phys Control Fusion 49, 1113 (2007).

[94] N. Joiner et al., Plasma Physics and Controlled Fusion 48, 685 (2006). 
[95] K. Matsuda, IEEE Transactions on Plasma Scence 17, 6 (1989).

[96] G. M. D. Hogeweij et al., Nuclear Fusion 38, 1881 (1998).

[97] M. R. D. Baar et al., Phys Plasmas 6, 4645 (1999).

[98] G. M. D. Hogeweij et al., Phys Rev Lett 76, 632 (1996).

[99] N. J. L. Cardozo et al., Plasma Physics and Controlled Fusion 39, B303 (1997).

[100] J. D. Callen, Phys Rev Lett 94, 055002 (2005).

[101] G. M. D. Hogeweij et al., Plasma Physics and Controlled Fusion 50, 065011 (2008).

[102] Y. V. Esipchuk et al., Plasma Physics and Controlled Fusion 45, 793 (2003).

[103] N. A. Kirneva et al., in Fusion Energy 2002 (Proc. 19th Int. Conf. Lyon, 2002 (Vienna: IAEA) CD-ROM file EX/P3-01 and http://www.iaea.org/programmes/ripc/physics/fec2002/html/fec2002.htm.

[104] V. A. Vershkov et al., Nuclear Fusion 45, S203 (2005).

[105] W. A. Houlberg, K. C. Shaing, and S. P. Hirshman, Physics of Plasmas 4, 3230 (1997).

[106] S. P. Hirshman, U. Schwenn, and J. Nührenberg, Journal of Computational Physics 87, 396 (1990).

[107] L. E. Zakharov and A. Pletzer, Physics of Plasmas 6, 4693 (1999).

[108] L. M. Degtyarev and V. V. Drozdov, Computer Physics Reports 2, 341 (1985).

[109] R. V. Budny, Nuclear Fusion 34, 1247 (1994).

[110] Équipe Tore Supra, presented by R Aymar, in Plasma Physics and Controlled Nuclear Fusion Research 1988 (Proc. 12th Int. Conf. Nice, 1988), Vol. 1, p. 9, IAEA, Vienna, 1989.

[111] B. Saoutic et al., Plasma Physics and Controlled Fusion 36B, 123 (1994).

[112] A. Becoulet et al., Physics of Plasmas 1, 2908 (1994).

[113] L. G. Eriksson, T. Hellsten, and U. Willen, Nuclear Fusion 33, 1037 (1993).

[114] G. T. Hoang et al., Phys Rev Letters 87, 125001 (2001).

[115] G. T. Hoang et al., Nuclear Fusion 38, 117 (1998).

[116] W. Horton et al., Phys Plasmas 7, 1494 (2000).

[117] A. Messiaen et al., Phys Plasmas 4, 1690 (1997).

[118] R. R. Weynants et al., Nuclear Fusion 39, 1637 (1999).

[119] C. Bourdelle et al., Nuclear Fusion 42, 892 (2002).

[120] M. Walters and C. M. Roach, Fusion Engineering and Design 71, 207 (2004).

[121] V. Basiuk et al., Nuclear Fusion 43, 822 (2003).

[122] G. Genacchi and A. Taroni, Jetto: A free boundary plasma transport code (basic version), Technical report, ENEA RT/TIB, 1988.

[123] M. Kotschenreuther, G. Rewoldt, and W. M. Tang, Computer Physics Communications 88, 128 (1995).

[124] C. Bourdelle et al., Nuclear Fusion 45, 110 (2005).

[125] C. M. Roach et al., Bulletin of the American Physical Society 47, 201 (2002).

[126] M. Honda and A. Fukuyama, Nuclear Fusion 46, 580 (2006).

[127] K. Itoh et al., Plasma Physics and Controlled Fusion 36, 279 (1994).

[128] H. Nordman et al., Nuclear Fusion 37, 413 (1997).

[129] R. E. Waltz et al., Phys Plasmas 4, 2482 (1997).

[130] M. Yagi et al., Journal of the Physics Society of Japan 66, 379 (1997).

[131] M. Erba et al., Plasma Physics and Controlled Fusion 39, 261 (1997).

[132] G. Bateman et al., Phys Plasmas 5, 1793 (1998).

[133] D. Hannum et al., Phys Plasmas 8, 964 (2001).

[134] T. Onjun et al., Phys Plasmas 8, 975 (2001).

[135] Y. N. Dnestrovskij et al., Plasma Physics Reports 30, 3 (2004).

[136] G. Bateman et al., Phys Plasmas 10, 4358 (2003).

[137] F. Porcelli, D. Boucher, and M. N. Rosenbluth, Plasma Physics and Controlled Fusion 38, 2163 (1996).

[138] B. B. Kadomtsev, Soviet Journal of Plasma Physics 1, 389 (1975).

[139] G. Bateman, C. N. Nguyen, and A. H. Kritz, Phys Plasmas 13, 072505 (2007).

[140] G. Becker and O. Kardaun, Nuclear Fusion 47, 33 (2007).

[141] G. Becker, Nuclear Fusion 35, 39 (1995).

[142] C. E. Singer et al., Computer Physics Communications 49, 275 (1988). 
[143] J. W. Connor et al., Nuclear Fusion 44, R1 (2004).

[144] P. Gohil et al., Nuclear Fusion 43, 708 (2003).

[145] J. E. Kinsey, G. M. Staebler, and R. E. Waltz, Phys Plasmas 12, 052503 (2005).

[146] T. Tala et al., Nuclear Fusion 46, 548 (2006).

[147] J. E. Kinsey et al., Nuclear Fusion 45, 450 (2005).

[148] TECHNICAL BASIS FOR THE ITER-FEAT OUTLINE, ITER EDA Documentation series 19, IAEA Vienna, 2000.

[149] ITER TECHNICAL BASIS, ITER EDA Documentation series 24, IAEA Vienna, 2002.

[150] Editor: J How, Project Integration Document (PID), Technical report, ITER Organisation, 2007.

[151] R. V. Budny et al., Nuclear Fusion 48, 075005 (2008).

[152] D. J. Campbell, Physics of Plasmas 8, 2041 (2001).

[153] S. C. Jardin, N. Pomphrey, and J. Delucia, Journal of Computational Physics 66, 481 (1986).

[154] C. E. Kessel et al., Nuclear Fusion 47, 1274 (2007).

[155] A. H. Kritz et al., Computer Physics Communications 164, 108 (2004).

[156] M. Sugihara et al., Plasma Physics and Controlled Fusion 45, L55 (2003).

[157] A. Pankin et al., Computer Physics Communications 164, 421 (2004).

[158] M. Valovic et al., Plasma Physics and Controlled Fusion 46, 1877 (2004).

[159] Y. Shimomura et al., Plasma Physics and Controlled Fusion 43, A385 (2001).

[160] A. R. Polevoi et al., Possibility of $Q>5$ stable, steady-state operation in ITER with moderate $\beta_{N}$ and H-factor, in Fusion Energy 2002 (Proc. 19th Int. Conf. Lyon, 2002) (Vienna: IAEA) CD-ROM file CT/P-08 and http://www.iaea.org/programmes/ripc/physics/fec2002/html/fec2002.htm.

[161] A. R. Polevoi, M. Shimada, and V. S. Mukhovatov, Plasma Physics and Controlled Fusion 48, A455 (2006).

[162] M. Sugihara et al., Nuclear Fusion 40, 1743 (2000).

[163] O. Sauter, C. Angioni, and Y. R. Lin-Liu, Physics of Plasmas 6, 2834 (1999).

[164] V. Mukhovatov et al., Nuclear Fusion 43, 942 (2003).

[165] D. E. Post et al., Atomic Data and Nuclear Data Tables 20, 397 (1977).

[166] Y. Murakami et al., Journal of Plasma Fusion Research 77, 712 (2001).

[167] A. R. Polevoi et al., Journal of Plasma Fusion Research 5, 82 (2002).

[168] D. Reiter et al., Plasma Physics and Controlled Fusion 33, 1579 (1991).

[169] A. S. Kukushkin et al., Journal of Nuclear Materials 337-339, 50 (2005).

[170] A. R. Polevoi et al., Nuclear Fusion 45, 1451 (2005).

[171] A. R. Polevoi et al., Nuclear Fusion 48, 015002 (2008).

[172] A. V. Zvonkov et al., Plasma Physics Reports 24, 389 (1998).

[173] A. R. Esterkin and A. D. Piliya, Nuclear Fusion 36, 1501 (1996).

[174] P. T. Bonoli et al., Benchmarking of Lower Hybrid Current Drive Codes with Application to ITER-Relevant Regimes, in Fusion Energy 2006 (Proc. 21st Int. Conf. Chengdu, 2006) (Vienna: IAEA) CD-ROM file IT/P1-2 and http://wwwnaweb.iaea.org/napc/physics/FEC/FEC2006/html/index.htm.

[175] R. Prater et al., Benchmarking of Codes for Electron Cyclotron Heating and Electron Cyclotron Current Drive under ITER Parameters, in Proceedings of the 14th Joint Workshop on Electron Cyclotron Emission and Electron Cyclotron Resonance Heating,Santorini, Greece, 2006. 